\title{
Fluctuations in Grandiose and Vulnerable Narcissistic States: A Momentary Perspective
}

\author{
by \\ Elizabeth A. Edershile \\ Bachelor of Arts, Washington University in St. Louis, 2014
}

Submitted to the

Kenneth P. Dietrich School of Arts \& Sciences in partial fulfillment of the requirements for the degree of

Master of Science

University of Pittsburgh 


\section{UNIVERSITY OF PITTSBURGH \\ DIETRICH SCHOOL OF ARTS AND SCIENCES}

This thesis was presented

by

\section{Elizabeth A. Edershile}

It was defended on

October 3, 2018

and approved by

Aidan G.C. Wright, Assistant Professor, Psychology

Stephen B. Manuck, Distinguished Professor, Psychology

Sarah L. Pedersen, Assistant Professor, Psychiatry

Thesis Advisor: Aidan G.C. Wright, Assistant Professor, Psychology 
Copyright (C) by Elizabeth A. Edershile 2019 


\title{
Fluctuations in Grandiose and Vulnerable Narcissistic States: A Momentary Perspective
}

\author{
Elizabeth A. Edershile, MS \\ University of Pittsburgh, 2019
}

It has been theorized that dynamic processes within and between grandiosity and vulnerability underlie pathological narcissism. Gaining an understanding of this process is quickly becoming one of the most pressing research goals in the study of narcissism. Research seeking to address this has either not studied both grandiosity and vulnerability together or has used dispositional measures to assess what are considered to be momentary states. Research in other areas of personality pathology (e.g., borderline personality disorder) has made headway in engaging data collection and analytic methods that are specifically meant to examine such questions. The present study took an exploratory approach to studying theorized fluctuations within and between grandiose and vulnerable states. Fluctuations were examined with regard to overall variability (measured by gross variability, instability, and inertia). Switching between discrete narcissistic states was also examined. Further, the present study examined connections between these momentary dynamics and dispositional narcissism assessments. Results suggest overall variability from moment to moment is moderately associated with dispositional assessments of narcissism. Specifically, individuals who are dispositionally grandiose appear to have high mean levels of both grandiosity and vulnerability and tend to vary in each. On the other hand, dispositionally vulnerable individuals tend to have high levels of vulnerability and low levels of grandiosity, though they do vary in each. Few results emerged with respect to switching between states. Future research should consider using similar methods and statistical techniques on different timescales to study theorized dynamics within narcissism. 
1.0 Introduction.................................................................................................................................................. 1

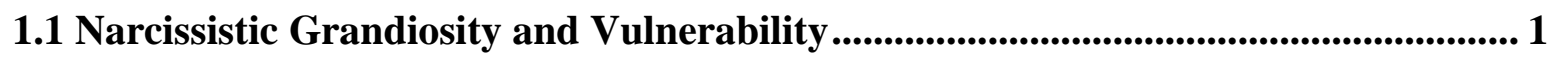

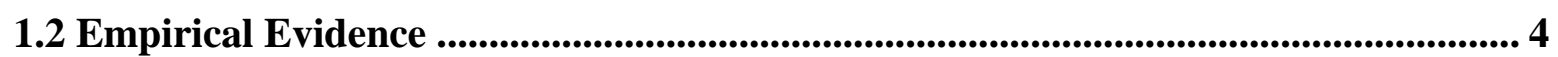

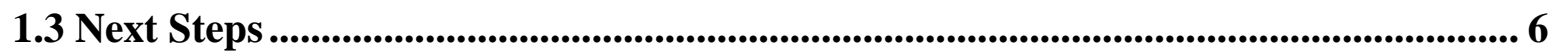

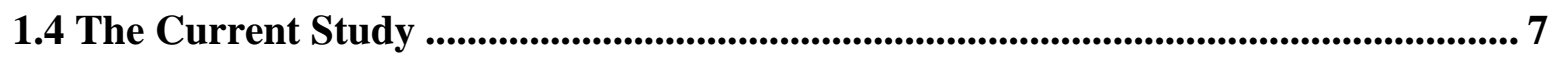

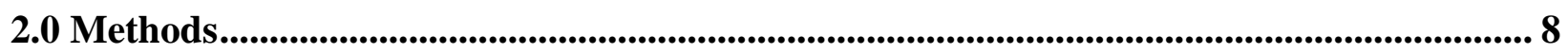

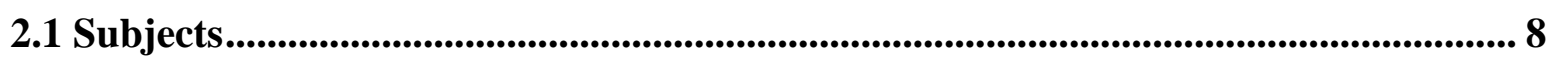

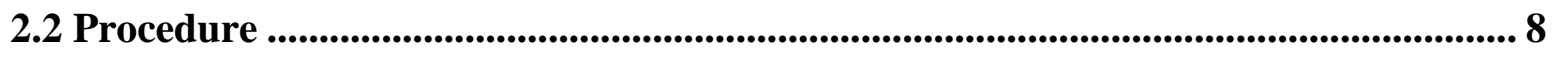

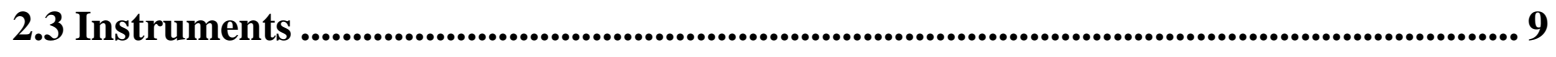

2.4 Data Analytic Plan......................................................................................................... 10

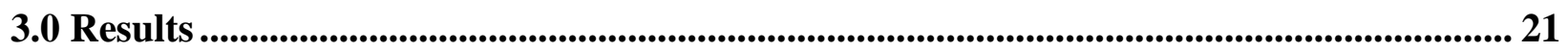

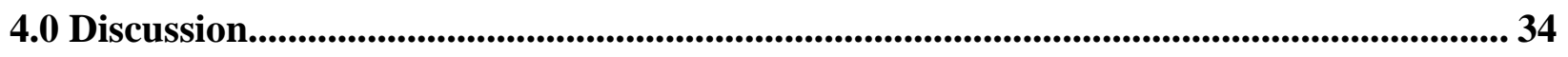

4.1 Limitations and Future Directions..................................................................... 42

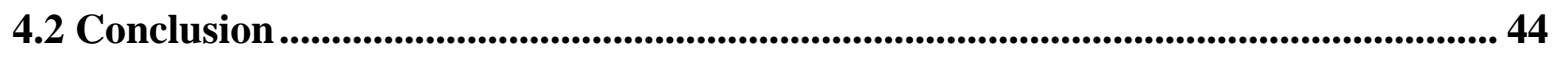

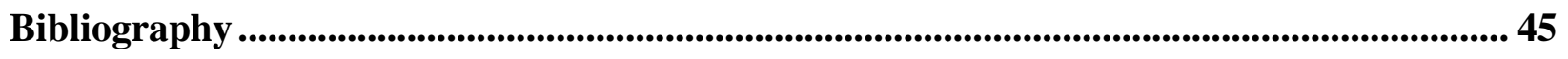




\section{List of Tables}

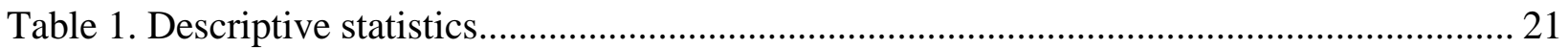

Table 2. Gross variability correlations among variables at the between person level................ 26

Table 3. Zero order and regression paths of instability at the between-person level.................. 27

Table 4. Results of multilevel regression results of inertia at the between person level ............. 28

Table 5. Switching propensity at the between-person level ............................................. 29

Table 6. Switching distance across switch and no switch for grandiosity and vulnerability........ 31

Table 7. Switching distance across switch and no switch for grandiosity and vulnerability with

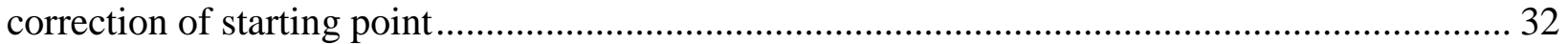




\section{List of Figures}

Figure 1. Proposed model of inertia at the between and within person level. NG = Narcissistic Grandiosity; NV = Narcissistic Vulnerability. 16

Figure 2. Raw scores of grandiosity for a subset of individual participants across time. 22

Figure 3. Raw scores of vulnerability for a subset of individual participants across time. 23

Figure 4. $\mathrm{N}=231$. Between-person path model using dispositional narcissism and mean level as predictors for average level variability across grandiosity and vulnerability. Doted lines represent associations between opposing narcissism variables (e.g., grandiosity).

Figure 5. $\mathrm{N}=231 . \mathrm{NG}=$ Narcissistic Grandiosity; NV= Narcissistic Vulnerability. Within-person results of inertia coefficients. Bolded values are those for which the credibility interval did not contain zero. Dotted lines are those for which the predicted domain. 28 


\subsection{Introduction}

Despite almost being removed from the Diagnostic and Statistical Manual of Mental Disorders - Fifth Edition (DSM-5; American Psychiatric Association, 2013), over the past decade, the study of narcissism has become increasingly popular with an average of 357 peer-reviewed articles published per year since 2010 (Miller, Lynam, Hyatt, \& Campbell, 2017). The recent interest in narcissism arose, in part, from a number of controversies regarding the ambiguity of construct's definition (Cain, Pincus, \& Ansell, 2008; Miller et al., 2017; Wright, 2014; 2016). Narcissism has enjoyed broad interest across the fields of clinical psychology, psychiatry, and social/personality psychology resulting in a large empirical literature that spans diverse areas of inquiry (Pincus \& Lukowitsky, 2010). Accordingly, definitions of narcissism have diverged across areas of study resulting in a fractured literature that can be difficult to integrate. The present research will focus on pathological narcissism. It is generally agreed that pathological narcissism is dimensional (e.g., Aslinger et al., 2018), and that its manifestations can be divided into two themes-narcissistic grandiosity and narcissistic vulnerability (Cain et al., 2008; Miller et al., 2017).

\subsection{Narcissistic Grandiosity and Vulnerability}

Central to theoretical articulations of narcissism are the contradictions within the construct.

How can a single coherent concept incorporate both grandiose and vulnerable components? Narcissistic grandiosity is defined by a grandiose sense of self, lack of empathy, and entitlement 
(e.g., Cain et al., 2008). Individuals high in narcissistic grandiosity are likely to be overtly immodest, self-promoting, and self-enhancing (Miller et al., 2017). Those same individuals are likely to endorse high levels of the basic personality traits of antagonism and extraversion (e.g., Paulhus \& Williams, 2002). Narcissistic grandiosity typically aligns well with a lay person's conceptualization of narcissism (Buss \& Chiodo, 1991).

Like narcissistic grandiosity, at the core of narcissistic vulnerability is entitlement and an intensely felt need for recognition. However, rather than manifesting in the overt reward seeking behavior of grandiosity, narcissistic vulnerability is associated with acute sensitivity to and avoidance of embarrassment and shame. Further, narcissistic vulnerability is characterized by selfdoubt, defensive social withdrawal, contingent self-esteem, and a constant need for external validation (e.g., Morf, 2006; Cain et al., 2008). Individuals high in narcissistic vulnerability are often distrustful of others and outwardly distressed and fragile (e.g., Miller et al., 2017). Narcissistic vulnerability is distinct from narcissistic grandiosity in that it is associated with pervasive negative emotionality and is broadly associated with other forms of personality pathology (Edershile, Simms, \& Wright, in press).

Until recently, the empirical literature has followed the conceptualization of narcissism set out by the $D S M$, primarily emphasizing grandiose features (e.g., Cain et al., 2008; Gabbard, 2009; Levy et al., 2007; Gore \& Widiger, 2016). For example, the most popular measure of narcissism to date is the Narcissistic Personality Inventory (NPI; Raskin \& Terry, 1988). The NPI was explicitly designed to capture the DSM-III definition of Narcissistic Personality Disorder (NPD), and thereby largely focused on the grandiose aspects of narcissism. As Cain and colleagues (2008) note, $77 \%$ of the research on narcissism during the years of 1985-2006 used the NPI as the primary measure of narcissism. One early exception is Wink's (1991) work, which included an analysis 
of six narcissism scales from the Minnesota Multiphasic Personality Inventory. Results indicated two largely unrelated components that he called Vulnerability-Sensitivity and GrandiosityExhibitionism. Following Wink's work, and despite the heavy reliance on a single measure of grandiose features (the NPI), interest in narcissistic vulnerability grew and it is now generally well accepted that both grandiosity and vulnerability reflect important constructs within the broader narcissism constellation (Pincus \& Lukowitsky, 2010). As such, there are a number of measures that have been developed that assess grandiosity, vulnerability, or both (e.g., Hyler, 1994; Back et al., 2013; Glover et al., 2012; Pincus et al., 2009).

Despite the fact that contemporary measures of grandiosity and vulnerability correlate, looking across the wide-range of available measures, each has a distinct pattern of antecedents, concurrent associations, and predictive validity (Miller, Dir, et al., 2010; Miller et al., 2014. 2016; Miller et al., 2017; Thomas et al., 2012). These results have been difficult to integrate and align with contemporary theoretical models. A potential contributor to this difficulty is that existing literature almost exclusively focused on narcissistic grandiosity and vulnerability as static individual differences (i.e., dispositional variables), whereas clinical theory and observation would suggest that these are best understood as dynamic processes occurring within the same individual over time. Trait measures developed for narcissism, with the aim of capturing relatively stable, trait-based descriptions, are in abundance. Using a battery of trait measures, as Miller and colleagues (2017) suggest, does not help us to understand the putative dynamic processes that link narcissistic grandiosity and vulnerability.

Although surface level descriptions and now voluminous empirical evidence suggest stark distinctions between grandiosity and vulnerability, clinicians have observed that both are often present within a single individual. As a result, it is likely that a dynamic within-person process, 
rather than exclusively static individual differences, governs their relationship. Case studies have been used to demonstrate the interplay of both grandiosity and vulnerability within an individual (e.g., Wright, 2014; Pincus, Cain, \& Wright, 2014). From these case studies, it is suggested that individuals with NPD do exhibit both grandiosity and vulnerability and, overtime, clinicians note a fluctuating pattern between the two. Empirical studies have lately begun to better understand and assess narcissistic grandiosity and vulnerability as they are described in the clinical literature.

Given that clinical observation and theory would suggest that grandiosity and vulnerability fluctuate within an individual over time, studies designed to capture these constructs as states, not just traits, and their within-person relationship are necessary next steps.

\subsection{Empirical Evidence}

Gore and Widiger (2016) were the first to systematically study whether characteristically grandiose or vulnerable individuals experience periods of vulnerability or grandiosity, respectively. Clinical psychology professors and clinicians were asked to think of someone they would classify as either a vulnerable narcissist or grandiose narcissist based on provided descriptions, and rate them on the core features of this description. They were subsequently asked to rate how often the individual exhibited the alternative narcissistic expression. For example, after rating someone they considered to be a grandiose narcissist, they were then asked to rate the individual on whether they ever exhibited vulnerable characteristics. Results indicated that individuals selected for exhibiting narcissistic grandiosity were particularly likely to also show vulnerable tendencies at some point. The opposite pattern, however, was not found to be true. Though this study did not directly examine patterns of fluctuation between grandiosity and 
vulnerability, or the degree to which each is present without the other, it does suggest that individuals selected for grandiose features will display both grandiose and vulnerable behavior at some point in time. These findings have subsequently been replicated by Hyatt and colleagues (2017).

In a wide-ranging review of the literature, Grubbs and Exline (2016) examined susceptibility of entitled individuals to experience vulnerability, or what the authors call "psychological distress." Their review suggested that entitled individuals may be particularly prone to experiencing vulnerability, or distress, due to their unrealistic expectations of their surrounding environment. Recall that entitlement is a central theme to both grandiosity and vulnerability (Miller et al., 2011; Dickinson \& Pincus, 2003). Grubbs and Exline (2016) suggest that when entitled individuals realize the mismatch between their expectations and reality, they will further try to bolster their self-esteem. That is, when in a state of vulnerable entitlement, narcissistic individuals will work towards a state of grandiose entitlement, which in turn perpetuates the cycle of entitlement, unmet expectations, and distress. This is in line with clinical theory, suggesting that narcissistic individuals shift between states of grandiosity and vulnerability. The specific question of how narcissistic grandiosity and vulnerability fluctuate, however, remains unanswered.

Some researchers have investigated the dynamics of narcissism using intensive longitudinal designs. Giacomin and Jordan (2016) examined daily ratings of narcissism and how within-person fluctuations of these ratings corresponded to daily life satisfaction and affect. This study measured participant's state ratings once a day for 14 days. The authors made a distinction between adaptive narcissism and maladaptive narcissism, such that maladaptive narcissism was associated with poorer psychological adjustments, more mood disorder symptoms, and lower self- 
esteem. The authors examined overall variability in each narcissism category. Overall, results revealed that variability in adaptive narcissistic features was associated with positive outcome variables (e.g., life satisfaction) whereas variability in maladaptive narcissistic features was associated with a poorer prognosis. Though not grandiosity and vulnerability, per se, a number of studies have examined self-esteem variability as it pertains to narcissism. Instability of self-esteem has often been referred to as a fragile self-esteem because it is thought to fluctuate between feelings of inadequacy and feelings of superiority (e.g., grandiose and vulnerable states; Akhtar \& Thomson, 1982; Rhodewalt \& Morf, 1995). Bosson and colleagues (2008) performed two small meta-analyses in which they reported on studies examining self-esteem variability in narcissism. No consistent pattern was found, such that some studies found that self-esteem variability and narcissism were related while others found no such pattern. More recently, however, Geukes and colleagues (2016) examined two facets of narcissistic grandiosity (admiration and rivalry) and their individual contributions to self-esteem level and variability. Results indicated that admiration was related to high and stable self-esteem, whereas rivalry was related to low and more variable self-esteem. Broadly, these results suggest there are specific patterns of variability with regard to narcissism. Though these studies are an important contribution to the literature on dynamic processes in narcissism, they do not directly assess the core features of grandiosity and vulnerability and each feature's pattern of variability.

\subsection{Next Steps}

Research seeking to understand the structure of narcissism, and specifically working to understand the clinically observed fluctuations of grandiosity and vulnerability, has begun to make 
progress. However, studies have either failed to study grandiosity and vulnerability directly (e.g., Geukes et al., 2016) or have continued to use trait measures to understand the dynamics of this relationships (e.g., Gore \& Widiger, 2016). As mentioned, dispositional assessments of narcissism have limited utility in understanding dynamic, state-level patterns. Future research calls for more advanced data capture and statistical techniques for assessing and modeling within-person dynamics of grandiosity and vulnerability.

\subsection{The Current Study}

Clinical theory has provided inadequate detail on how fluctuations in narcissism occur. Information on the specific timescale and patterning (e.g., order) of these manifestations has not been systematically examined. Broadly, I hypothesize that grandiosity will be associated with relative stability whereas vulnerability will be associated with relative instability. I will use dispositional measures of narcissism as predictors for different forms of fluctuations. This allows me to investigate how those higher in narcissism vary across time compared to those lower in dispositional narcissism. The goal of the present study is to systematically evaluate patterns of fluctuation in narcissistic states, and potentially demonstrate the need to augment static measures of NPD with work on dynamic measures and research designs that uncover the mechanisms at play in NPD. I believe that shifting this focus will result in a more nuanced and accurate picture of narcissism. 


\subsection{Methods}

\subsection{Subjects}

Undergraduates $(N=231)$ from the University of Pittsburgh were recruited from the Psychology Department Subject Pool to take part in this study. Participants completed study procedures in exchange for two course credits. Participants had to be 18 years of age and had to own an up to date smartphone (i.e., purchased within in the last 3 years and with up to date software). This sample was somewhat more male than female (61\% and $38.5 \%$, respectively; one

declined to answer). Ages ranged from 18 to $26(M=18.82, S D=1.08)$. The majority of participants identified as Caucasian (69.3\% White; $16.9 \%$ Asian; 5.6\% Black; $8.2 \%$ multiracial)

\subsection{Procedure}

Participants came to an on-campus computer lab for training and assessment in groups of 20-30 participants. Participants were briefed on procedures and given a battery of self-report measures via the computer. After completing the in-person assessments, participants were trained on using the software that was installed on their smartphone for one week.

For the ambulatory assessment portion of the study, compliance was high $(7,480$ total entries). Participants completed up to 42 assessments $(M=32.38 ; S D=7.99)$ over the course of the week (maximum of six surveys per day) between 10:00 and 22:00 each day. Surveys were designed to appear at random times throughout the day and participants were prompted when a 
new survey was available. Once prompted to complete a survey, participants had 30 minutes to fill out the survey on the smartphone. Each assessment took 3-5 minutes to complete. Full credit was given if participants completed $50 \%$ of the random surveys. Participation beyond this minimum amount was incentivized with random drawings for additional rewards (Apple watch, Nintendo Switch, Play Station 4), with chances of winning proportional to amount of participation.

\subsection{Instruments}

\section{The Pathological Narcissism Inventory (PNI; Pincus et al., 2009)}

Trait level Narcissistic vulnerability and grandiosity was assessed using the 28 -item brief form (Schoenleber et al., 2015). It was developed to assess pathological narcissistic traits. The questionnaire measures narcissistic grandiosity (Exploitativeness, Grandiose Fantasy, Selfsacrificing Self-Enhancement) and narcissistic vulnerability (Contingent Self-esteem, Hiding the Self, Entitlement Rage, Devaluing; Wright, Lukowitsky, Pincus, \& Conroy, 2010). The PNI is a frequently used measure that has strong support for its validity (e.g., Morf et al., 2017; Wright et al., 2013; Houlcroft et al., 2012). All items are scored on a 6-point Likert scale (0, not at all; 5, very much like me). Global measures of grandiosity and vulnerability were computed by averaging the items that make up each of the subscales. Internal consistency for the PNI was adequate (Grandiosity $\alpha=.82$; Vulnerability $\alpha=.86$ ).

Narcissistic Grandiosity Scale (NGS; Crowe et al., 2016; Rosenthal et al., 2007)

State level narcissistic grandiosity was assessed using the NGS. This measure consists of a set of 16 adjectives that assess narcissistic grandiosity. An unpublished factor analysis was previously completed with University of Pittsburgh students. From this, four out of these 16 
adjectives with the highest factor loadings on grandiosity were selected for the present study. These four adjectives were Glorious, Prestigious, Brilliant, and Powerful. These items were administered as part of the EMA survey with a 100-point sliding scale in which Not at all and Extremely were anchors. Reliability of the NGS was adequate $\left(\alpha_{\text {within }}=.80 ; \alpha_{\text {between }}=.98\right)$.

Narcissistic Vulnerability Scale (NVS; Crowe et al., 2018)

This measure consists of a set of 12 adjectives thought to assess narcissistic vulnerability and is meant as a complementary measure to the NGS. Similar to above, an unpublished factor analysis revealed four adjectives with strongest loadings on vulnerability: Underappreciated, Misunderstood, Ignored, and Resentful. These four adjectives were used in the current study. As above, these four items were administered as part of the EMA survey with a 100-point sliding scale in which Not at all and Extremely were anchors. Reliability of the NVS was adequate ( $\alpha_{\text {within }}$ $\left.=.77 ; \alpha_{\text {between }}=.96\right)$.

\subsection{Data Analytic Plan}

\section{Overall Variability}

Aim one: To establish whether, and the degree to which, grandiosity and vulnerability each independently vary moment to moment within persons.

To first establish whether variability in grandiosity and vulnerability exists, the current study followed a conceptually similar analytic approach to Houben and colleagues (2015; see also Wang et al., 2012). Using such techniques allowed for the examination of (i) overall variability in narcissism ratings across time (gross variability), (ii) the degree to which individuals are likely to shift markedly in state ratings from one state to the next (instability), and (iii) the amount of carry- 
over from one state to the next (inertia). Given that dispositional narcissism measures have been the primary method on which our knowledge of narcissism is based, and will continue to enjoy widespread use moving forward, dispositional narcissism scores (i.e., PNI) were used as predictors of individual differences in gross variability, instability, and inertia to determine whether these vary as a function of dispositional narcissism levels.

\section{Gross Variability}

Gross variability is a summary statistic of the range of an individual's states across time. Gross variability can be estimated by calculating each individual's within-person standard deviation (iSD) of grandiosity and vulnerability across time. However, I adopted a multi-level modeling approach to evaluating gross variability, as it allows for each participant's contribution to be weighted by how many responses they contributed. In other words, someone who only completed 4 entries will not have as reliable of a gross variability score as someone who had a complete set of 42 responses. Following Geukes and colleagues (2016), who used a similar approach in the study of self-esteem variability, I used multilevel models that relax the assumption of homogeneous level 1 (i.e., time-varying) residuals, and allow for predictors of individual differences in momentary variation. The higher-order PNI scores of grandiosity (PNI-G) and vulnerability (PNI-V) were used as predictors of individual differences in momentary variability. The model was specified as follows (the example is given with momentary grandiosity, but was repeated with vulnerability),

Level 1: Grandiosity ${ }_{\mathrm{ti}}=\beta_{0 \mathrm{i}}+\varepsilon_{\mathrm{ti}}$

Level 2: $\beta_{0 \mathrm{i}}=\gamma_{00}+\gamma_{01}\left(\mathrm{PNI}-\mathrm{G}_{\mathrm{i}}\right)+\gamma_{02}\left(\mathrm{PNI}-\mathrm{V}_{\mathrm{i}}\right)+u_{0 \mathrm{i}}$

$\sigma^{2}{ }_{\mathrm{i}}=\exp \left(\gamma_{10}+\gamma_{11}\left(\mathrm{PNI}_{-\mathrm{G}_{\mathrm{i}}}\right)+\gamma_{12}\left(\mathrm{PNI}-\mathrm{V}_{\mathrm{i}}\right)+\gamma_{13}\left(\overline{\text { Grandiosity }_{l}}\right)+\right.$ $\left.\gamma_{14}(\overline{\text { Vulnerabllity }})+u_{1 \mathrm{i}}\right)$ 


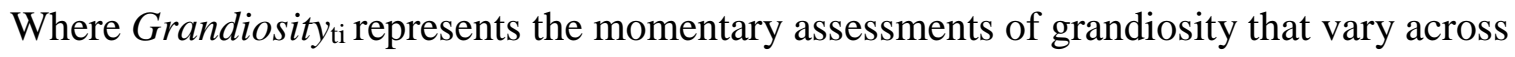
time (subscript $\mathrm{t}$ ) and individuals (subscript $\mathrm{i}$ ), $\beta_{0 \mathrm{i}}$ represents the random intercept that varies across individuals, $\varepsilon_{\mathrm{ti}}$ reflects the momentary departures in grandiosity from each individuals intercept across time and participants, $\gamma_{00}$ reflects the grand intercept or expected value when PNI-G and PNI-V are at the mean, $\gamma_{01}$ is the effect of PNI-G on individual differences in momentary grandiosity, $\gamma_{02}$ is the effect of PNI-V on individual differences in momentary grandiosity, and $u_{0 \mathrm{i}}$ reflects the randomly varying residuals in intercepts. Multilevel models typically assume that the variance of within-person residuals (i.e., $\sigma_{\text {ti) }}^{2}$ is constant across individuals. However, here I relaxed that assumption and $\sigma_{\text {ti }}^{2}$ was allowed to vary across individuals and as a function of PNI scores. Values of variances need to be positive, which is achieved by using an exponential function when modeling the variance. Here $\gamma_{10}$ represents the average variability score when PNI-G and PNI-V are $0, \gamma_{11}$ is the effect of PNI-G on individual differences in variability, $\gamma_{12}$ is the effect of PNI-V on individual differences in variability, $\gamma_{13}$ is the effect of the mean of grandiosity on individual differences in variability, $\gamma_{14}$ is the effect of the mean of vulnerability on individual differences in variability, and $u_{1 \mathrm{i}}$ reflects residual individual differences in variability.

Currently there is a debate in the literature about how associations between individual differences in variability and within-person means should be interpreted. Many argue that positive associations between within-person means and variability merely reflect that people with higher means have more room to vary, and thus this relationship is artifactual (e.g., Baird, Le, \& Lucas, 2006). In particular, it is argued that individual means and standard deviations are associated due to floor or ceiling effects that artificially constrain the variance for individuals close to the boundary. Baird and colleagues (2006) demonstrated that even when means and standard deviations are independent, when distributions are skewed (as is often the case with narcissism 
variables), associations between the means and standard deviations become an artifact of the analyses. As a result, it is possible that associations between variability and predictors (e.g., PNI scores) may be due to trait-level variance. Given this, I included between-person mean levels of grandiosity and vulnerability as covariates and examined the relationship between PNI scores and variability adjusting for the mean. However, I also examined zero-order correlations between all variables to fully understand the associations among the variables.

Specific Hypothesis: Higher PNI-G scores would be related to more stability in both state level grandiosity and vulnerability. Higher PNI-V scores would be related to more variability in both state level grandiosity and vulnerability. Each would be related to their momentary counterparts (e.g., PNI-G predicted individual differences in momentary assessed grandiosity). Overall mean levels would be associated with their matched PNI scores and their matched overall variability.

\section{Instability}

Whereas gross variability summarizes the dispersion in scores without considering temporal ordering, instability is a metric that summarizes the magnitude of change from one moment to the next. Instability is often calculated as each individual's mean squared successive differences (iMSSD) between consecutive narcissism scores. However, I adopted a multi-level modeling framework to examine instability. PNI-G and PNI-V were used as predictors of individual differences in squared successive differences (SSD) in grandiosity (SSD-G) and vulnerability (SSD-V). The model was specified as follows (again, the example is given with SSD-G but was repeated with SSD-V).

Level 1: $S S D-G_{\mathrm{ti}}=\beta_{0 \mathrm{i}}+\varepsilon_{\mathrm{ti}}$

Level 2: $\beta_{0 \mathrm{i}}=\gamma_{00}+\gamma_{01}\left(\mathrm{PNI}-\mathrm{G}_{\mathrm{i}}\right)+\gamma_{02}\left(\mathrm{PNI}-\mathrm{V}_{\mathrm{i}}\right)+u_{0 \mathrm{i}}$ 
Where $S S D-G_{\mathrm{ti}}$ represents the SSD for grandiosity that vary across time (subscript t) and individuals (subscript i), $\beta_{0 \mathrm{i}}$ represents the random intercept of SSD-G that varies across individuals, $\varepsilon_{\mathrm{ti}}$ reflects the momentary departures in SSD-G from each individuals intercept across time and participants, $\gamma_{00}$ reflects the grand intercept or expected value of SSD-G when PNI-G and PNI-V are $0, \gamma_{01}$ is the effect of PNI-G on individual differences in SSD-G, $\gamma_{02}$ is the effect of PNI$\mathrm{V}$ on individual differences in SSD-G, and $u_{0 \mathrm{i}}$ reflects the randomly varying residuals in intercepts.

Specific Hypothesis: Higher PNI-G scores would be related to lower SSD-G and SSD-V values while higher PNI-V scores would be related to higher SSD-G and SSD-V values.

\section{Inertia}

Inertia is a metric that quantifies the degree to which a previous state predicts the current state. Accordingly, it indicates how quickly a person returns to baseline after being perturbed. In other words, inertia can be understood as how well a person is able to regulate themselves. In most psychological data, the value ranges between 0 and 1 , and the closer it is to 1 , the longer it takes a person to return to his/her baseline. An individual with high grandiose inertia has grandiose states that are more self-predictive across time, and which tend to ramp up and diminish more slowly over time. Conversely, an individual who is prone to unpredictable oscillations will have a lower inertia than someone who either stays constant from one point to the next or someone who predictably fluctuates between states. I calculated inertia in a multi-level modeling framework with PNI-G and PNI-V as predictors of individual differences in state-level ratings of grandiosity and vulnerability (see Figure 1). Dynamic Structural Equation Modeling (DSEM; e.g., Asparouhov et al., 2017, 2018) was used to simultaneously calculate lagged narcissism values (e.g., $\mathrm{G}_{\mathrm{t}-\mathrm{i}}$ ) as well as perform the multilevel model. Though models were run for both grandiosity and vulnerability, only grandiosity is shown below. 
Level 1: Grandiosity ${ }_{\mathrm{ti}}=\beta_{0 \mathrm{i}}+\beta_{1 \mathrm{i}}\left(\mathrm{G}_{\mathrm{t}-1 \mathrm{i}}\right)+\varepsilon_{\mathrm{ti}}$

Level 2: $\beta_{0 \mathrm{i}}=\gamma_{00}+\gamma_{01}\left(\mathrm{PNI}-\mathrm{G}_{\mathrm{i}}\right)+\gamma_{02}\left(\mathrm{PNI}-\mathrm{V}_{\mathrm{i}}\right)+u_{0 \mathrm{i}}$

$$
\beta_{1 \mathrm{i}}=\gamma_{10}+\gamma_{11}\left(\mathrm{PNI}-\mathrm{G}_{\mathrm{i}}\right)+\gamma_{12}\left(\mathrm{PNI}-\mathrm{V}_{\mathrm{i}}\right)+u_{l \mathrm{i}}
$$

Where Grandiosity $y_{\mathrm{ti}}$ represents the momentary assessments of grandiosity that vary across time (subscript $\mathrm{t}$ ) and individuals (subscript $\mathrm{i}$ ), $\beta_{0 \mathrm{i}}$ represents the random intercept that varies across individuals, $\beta_{1 \mathrm{i}}\left(\mathrm{G}_{\mathrm{t}-\mathrm{li}}\right)$ represents the effect of grandiosity at the previous time point (t-1) that varies across individuals (i.e., a random slope), $\varepsilon_{\mathrm{ti}}$ reflects the momentary departures in grandiosity from each individual's intercept across time and participants, $\gamma_{00}$ reflects the grand intercept or expected value of grandiosity when PNI-G and PNI-V are 0 and an individual's mean grandiosity at t-1, $\gamma_{01}$ is the effect of PNI-G on individual differences in momentary grandiosity, $\gamma_{02}$ is the effect of PNI$\mathrm{V}$ on individual differences in momentary grandiosity, and $u_{0 \mathrm{i}}$ reflects the randomly varying residuals in intercepts, $\gamma_{10}$ reflects the average effect of grandiosity at t- 1 when PNI-G and PNI$\mathrm{V}$ are $0, \gamma_{11}$ is the effect of PNI-G on individual differences in inertia, $\gamma_{12}$ is the effect of PNI-V on inertia, and $u_{l \mathrm{i}}$ reflects the randomly varying residuals in slopes.

Specific Hypothesis: Higher PNI-G scores would be related to higher inertia values for both state level grandiosity and vulnerability. Higher PNI-V scores would be related to lower inertia values for both state level grandiosity and vulnerability.

General Hypothesis: Higher PNI-G scores would be related to more stability in state-level grandiosity and vulnerability, as measured by gross variability, instability, and inertia, while higher PNI-V scores would be related to greater fluctuations in state-level grandiosity and vulnerability. 
Decomposition Within-Person
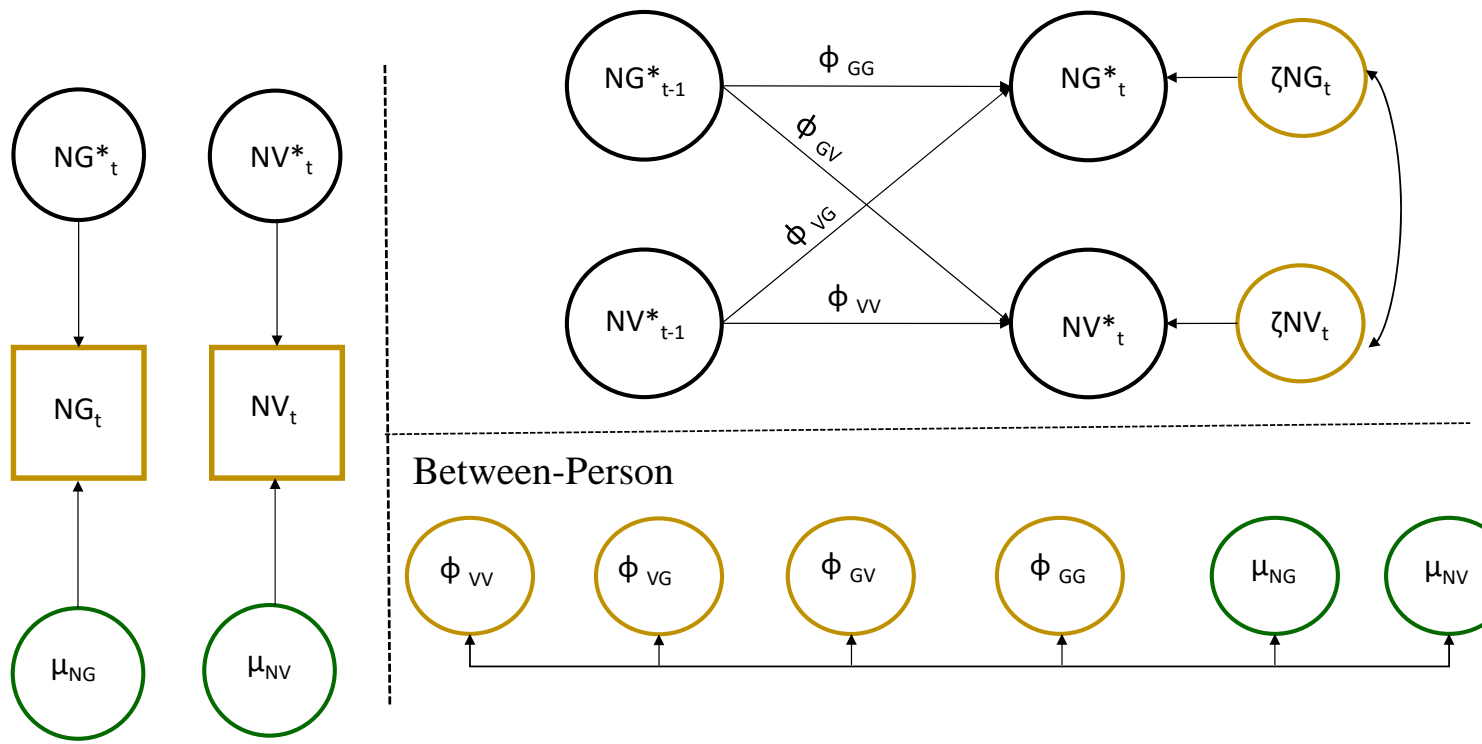

Between-Person

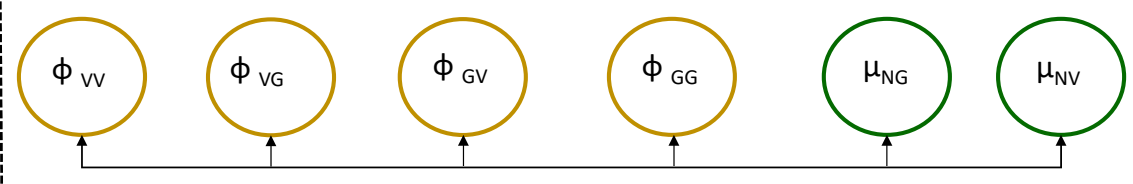

Figure 1. Proposed model of inertia at the between and within person level. NG = Narcissistic Grandiosity; NV = Narcissistic Vulnerability.

\section{Switching}

Aim two: Examine the variability between states (i.e., switching) of grandiosity and vulnerability within an individual.

Once it was established that overall variability existed (accomplished by examining gross variability, instability, and inertia), I examined whether fluctuations between states occurred. That is, do individuals actually vacillate between states of grandiosity and vulnerability in a dynamic and predictable fashion? Houben and colleagues (2016) examined fluctuations, or what the authors refer to as "switching", between positive and negative emotional states in individuals with borderline personality disorder (BPD) compared to healthy controls. I followed similar analytic techniques to answer questions such as (i) whether individuals are more likely to switch from a grandiose state to a vulnerable one or vice versa, (ii) whether individuals with higher narcissism 
scores are more likely the switch between states of grandiosity and vulnerability, and (iii), whether individuals with higher narcissism scores have greater extremes in their switching (e.g., from a more grandiose state to a more vulnerable state).

A state was labeled as grandiose when an individual was above the grand mean for grandiosity and below the grand mean for vulnerability. The reverse was true for a vulnerable state. A state was labeled as neither grandiose nor vulnerable if an individual was below the mean in both grandiosity and vulnerability ratings. A state was labeled as mixed if an individual was above the mean in both grandiosity and vulnerability. For all switching analyses, the first narcissism rating of every day was set as missing, such that overnight changes were never calculated.

\section{Switching Propensity}

Switching propensity refers to the likelihood of switching between states. For each pair of consecutive vulnerability and grandiosity ratings, I coded whether a switch occurred or not $(\mathrm{SWITCH}=1 ; \mathrm{NON}-\mathrm{SWITCH}=0$, respectively). The next step was to examine the propensity to switch from grandiose to vulnerable, specifically, and vice versa. I also examined the propensity to switch within a given domain (e.g., grandiosity). The same dummy coding pattern was used to examine switching above threshold to below and vice versa within both domains. Additionally, I examined whether switching propensity was related to dispositional narcissism scores. Two-level logistic regression models were used in which the probability to switch was modeled at level 1. Switching (either present or absent) was examined as a function of dimensional endorsement of PNI scales in model 2.

Level 1:

$\operatorname{Prob}\left(\right.$ Switch $\left._{\mathrm{ti}}=1 \mid \beta_{\mathrm{i}}\right)=\phi_{\mathrm{ti}}$ 
$\log \left[\phi_{\mathrm{ti}} /\left(1-\phi_{\mathrm{ti}}\right)=\eta_{\mathrm{ti}}\right.$

$\eta_{\mathrm{ti}}=\beta_{0 \mathrm{i}}$

Level 2:

$\mathrm{B}_{0 \mathrm{i}}=\gamma_{00}+\gamma_{01} \mathrm{PNI}-\mathrm{G}_{\mathrm{i}}+\gamma_{02} \mathrm{PNI}-\mathrm{V}_{\mathrm{i}}+u_{0 \mathrm{i}}$

A $\log$ link is used to model switching as a function of narcissism endorsement. Where $\beta_{0 \mathrm{i}}$ is a random intercept that varies across individuals, $\gamma_{00}$ is the mean log-odds to switch when PNIG and PNI-V are 0, $\gamma_{01}$ is the effect of PNI-G on switching propensity, $\gamma_{02}$ is the effect of PNI-V on switching propensity, and $u_{0 \mathrm{i}}$ is the randomly varying residuals in intercepts.

Specific Hypothesis: Higher PNI-V scores would be related to a higher probability to switch. Higher PNI-G scores would be related to a lower probability to switch.

\section{Switching Distance}

Not only is it important to understand switching from one state to the next, but it's also worthwhile to examine switching with respect to magnitude of change. This can only be done for within-domain switches, rather than cross-domain switches. Two-level regression models were used in which switch and non-switch states were used as predictors of absolute consecutive changes in valence (level of grandiosity and vulnerability) at level 1. In level 2, dimensional PNI features were used to estimate the slopes of absolute consecutive changes from model 1.

Level-1 model:

In $(\text { absolute consecutive difference })_{\mathrm{ti}}=\beta_{1 i} \mathrm{SWITCH}_{t i}+\beta_{2 i} \mathrm{NO}_{-} \mathrm{SWITCH}_{t i}+r_{t i}$ Level-2 model:

$\beta_{1 i}=\gamma_{10}+\gamma_{11} \mathrm{PNI}-\mathrm{G}_{\mathrm{i}}+\gamma_{12} \mathrm{PNI}-\mathrm{V}_{\mathrm{i}}+u_{1 i}$ 


$$
\beta_{2 i}=\gamma_{20}+\gamma_{21} \mathrm{PNI}-\mathrm{G}_{\mathrm{i}}+\gamma_{22} \mathrm{PNI}-\mathrm{V}_{\mathrm{i}}+u_{2 i}
$$

At level 1, the absolute difference between consecutive narcissism ratings is modeled as a function of whether a switch occurred $(\mathrm{SWITCH}=1)$ or not $(\mathrm{NO}$ _SWITCH=1), excluding the intercept from the model. At level 2, $\beta_{1 i}$ represents the subject-specific average total difference between consecutive narcissism ratings for switches, $\beta_{2 i}$ presents subject-specific average total difference between consecutive narcissism ratings for nonswitches, $\gamma_{10}$ and $\gamma_{20}$ reflect the grand intercept or expected value in absolute changes when PNI-G and PNI-V are $0, \gamma_{11}$ and $\gamma_{21}$ are the effect of PNI-G on individual differences in switching and non-switching distance, respectively, $\gamma_{12}$ and $\gamma_{22}$ are the effect of PNI-V on individual differences in switching and non-switching distance, respectively, and $u_{1 i}$ and $u_{2 i}$ reflect the randomly varying residuals in intercepts.

Specific Hypothesis: Higher PNI-V scores would be related to an overall greater magnitude of change. Higher PNI-G scores would be related to an overall smaller magnitude of change.

\section{Switch Distance with Correction for Starting Point of Switch}

Given that switching distance may be related to the individual's starting point, whether switch distance is driven by differences in starting points was examined. To do this, I used the same aforementioned multilevel modeling techniques used in analyzing switching distance but included the level of grandiosity and vulnerability rating at one time point prior to previous analyses (i.e., $t$-1; the starting point of each calculated distance). This form of centering allowed me to hold the starting point for each distance constant at the average for all participants. 
Specific Hypothesis: Higher PNI-V scores would continue to be related to greater switching distance when holding the starting point constant. Higher PNI-G scores would continue to be related to smaller switching distance when holding the starting point constant.

\section{Switch Distance to Different Directions}

The final step is to examine switching distance for higher PNI-G scores and higher PNI-V scores when examining switching to a specific direction (e.g., switches from above threshold in a

grandiose state to below). Again, two-level regression models were used to examine absolute differences between consecutive state ratings as a function of a switch to grandiose, a switch to vulnerable, and no switching. In model 2, dimensional PNI features were used to predict slopes from model 1.

Specific Hypothesis: Higher PNI-V scores would be related to a greater likelihood to switch from a vulnerable state to a grandiose state, but the reverse would not necessarily be true (i.e., higher PNI-G scores would not be related to a greater likelihood to switch from a grandiose to a vulnerable state).

General Hypothesis: Higher PNI-V scores would be related to a greater likelihood to switch between states. Higher PNI-G scores would be related to a lower likelihood to switch between states. 


\subsection{Results}

All results were run in Mplus Version 8.1 (Muthén \& Muthén, 1998-2018). All analyses were run with the Bayesian Estimator function in Mplus. Due to the large number of results, a summary will be presented in text. For complete results, please refer to the tables and figures. Means and standard deviations of all measures can be found in Table 1.

Table 1. Descriptive statistics

\begin{tabular}{lcr}
\hline & Mean & SD \\
\cline { 2 - 3 } PNI Grandiosity & 1.9 & .5 \\
PNI Vulnerability & 1.7 & .6 \\
& & \\
Momentary Grandiosity & 25.9 & 23.9 \\
Momentary Vulnerability & 16.5 & 18.4 \\
\hline $\begin{array}{l}\text { Note. N }=231 . \text { SD }=\text { Standard Deviation; PNI }=\text { Pathological } \\
\text { Narcissism Inventory. }\end{array}$ &
\end{tabular}

As an initial presentation of overall variability, raw scores for a subset of individuals across time can be found in Figure 2 for grandiosity and Figure 3 for vulnerability. As shown graphically, participants appear to vary in substantially different ways. Of note, the intraclass correlation (ICC) for momentary grandiosity is .74 and the ICC for momentary vulnerability is .65 . Nonetheless, there remains substantial within-person variability. 


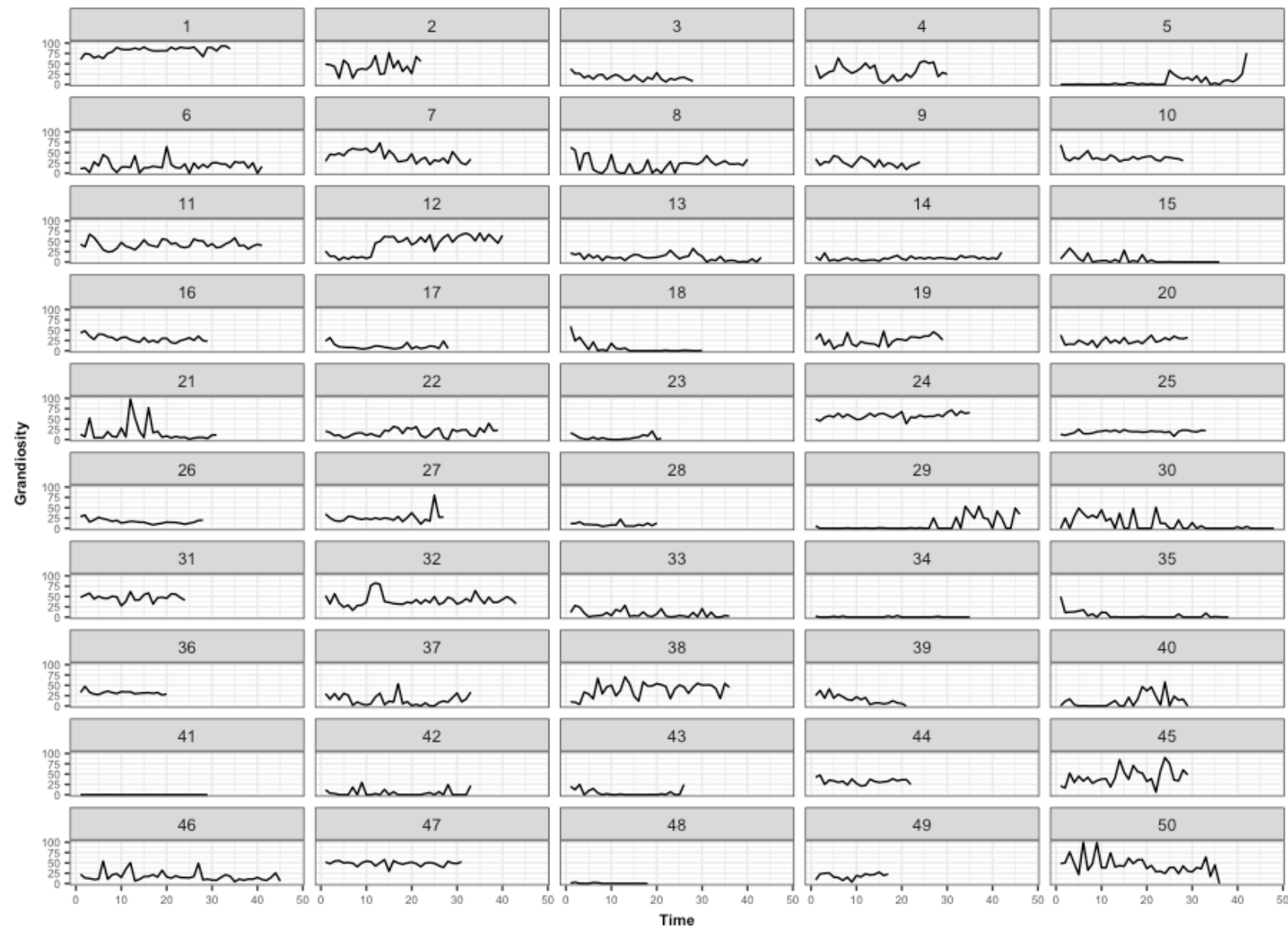

Figure 2. Raw scores of grandiosity for a subset of individual participants across time. 


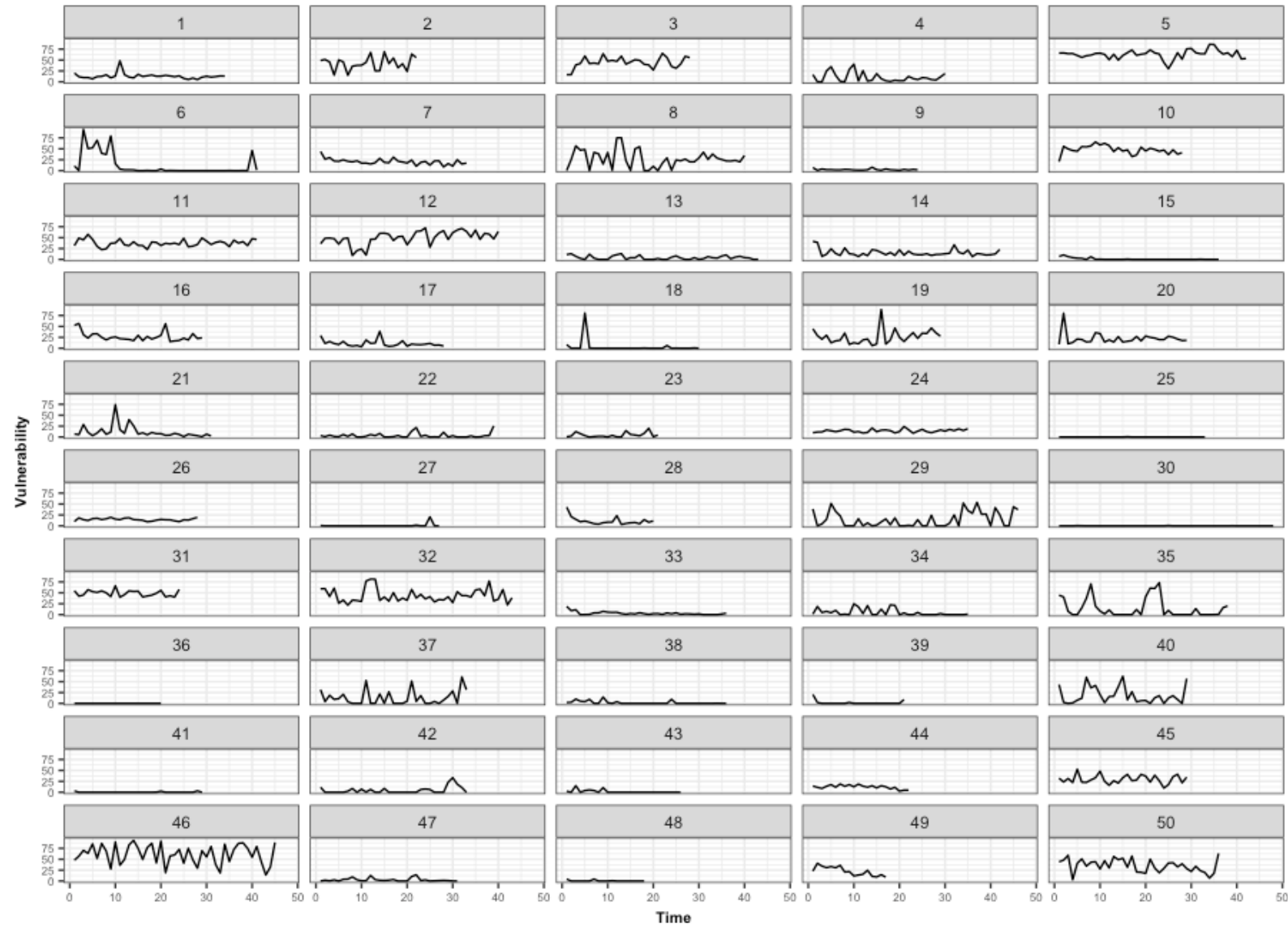

Figure 3. Raw scores of vulnerability for a subset of individual participants across time.

\section{Gross Variability}

Table 2 shows the correlations among all variables. Figure 4 indicates associations among variables, once accounting for the mean of momentary grandiosity and vulnerability. In both correlations and regression models, within-person mean levels tended to be moderately associated with gross variability in the matched dimension (e.g., within-person mean of grandiosity and variability in grandiosity). For both domains, somewhat smaller correlations were observed across domains between within-person means and variability. These associations were reduced in the 
regression models.

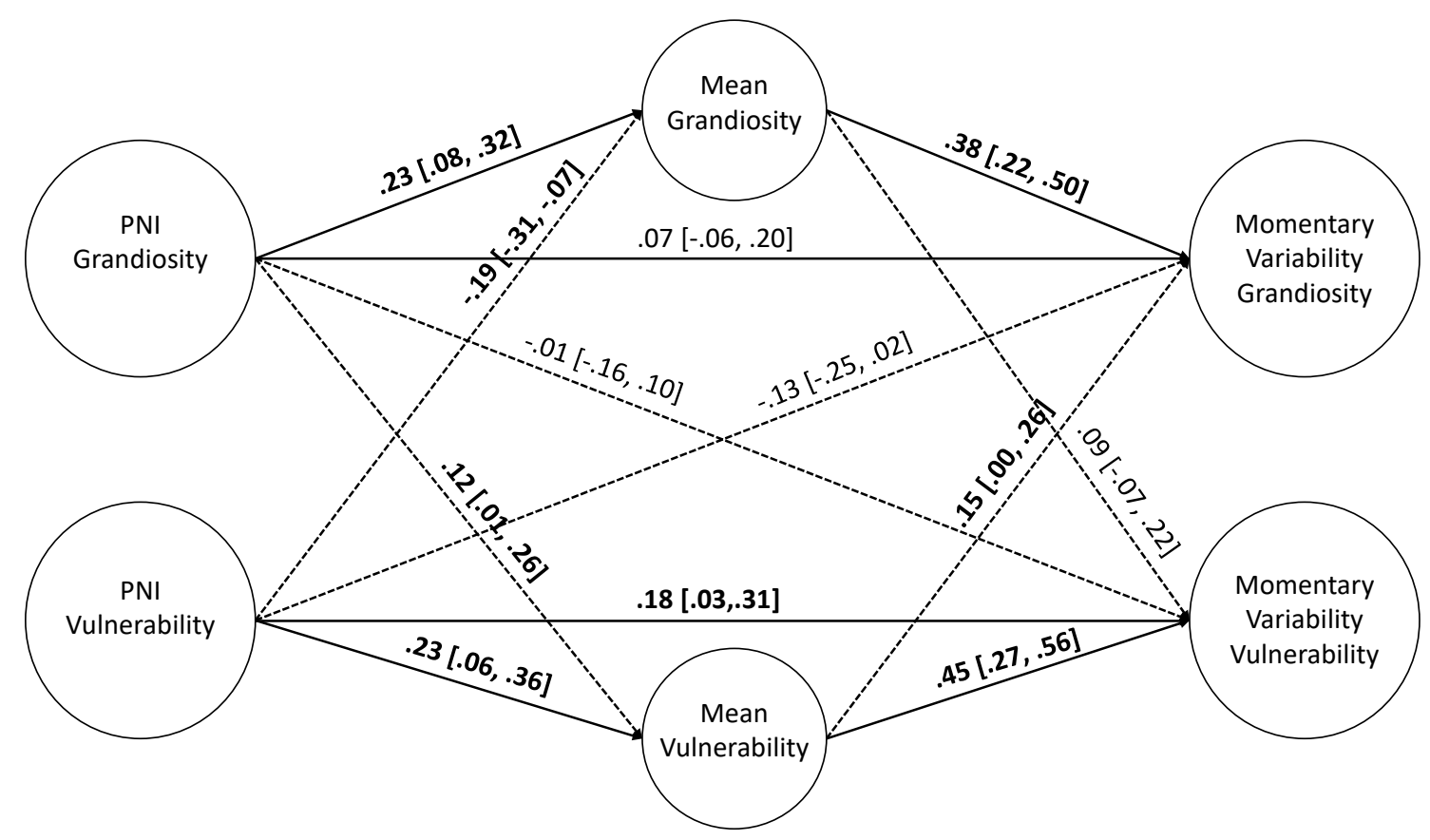

Figure 4. $\mathbf{N}=231$. Between-person path model using dispositional narcissism and mean level as predictors for average level variability across grandiosity and vulnerability. Doted lines represent associations between opposing narcissism variables (e.g., grandiosity).

Dispositional scores were modestly correlated with same domain within-person means, and effect sizes were similar once accounting for shared variance in disposition scores in the regression models. Dispositional grandiosity was a modest but significant predictor of within-person means of vulnerability, whereas dispositional vulnerability predicted lower mean levels of momentary grandiosity. Dispositional grandiosity scores were modestly and non-significantly associated with gross variability in grandiosity in both correlational and regression analyses. Dispositional vulnerability scores were significantly associated with variability in vulnerability, with a moderate correlation and modest regression path. Moving to cross-domain associations, dispositional grandiosity was modestly correlated with variability in vulnerability but unassociated in the 
regression model, whereas vulnerability was unassociated with gross variability in grandiosity in correlations and regressions. 
Table 2. Gross variability correlations among variables at the between person level

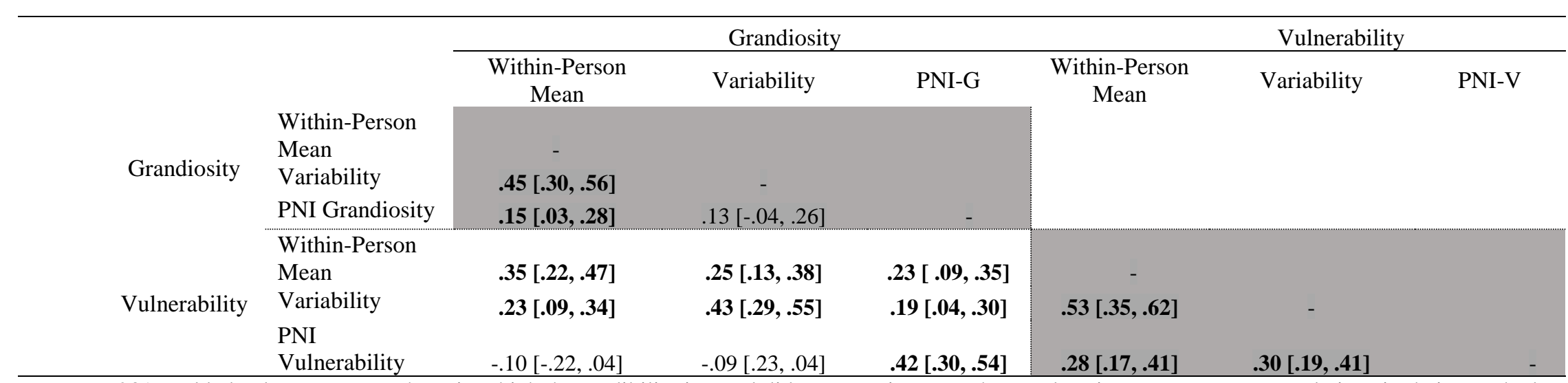

Note. $N=231$. Bolded values represent those in which the credibility interval did not contain zero. Those values in gray represent correlations in their matched narcissism category. 


\section{Instability}

At the within-person level, Squared Successive Difference (SSD) scores of grandiosity and vulnerability were correlated at $r=.11$. At the between-person level, these variables were correlated at $r=.47$ in the regression model and $r=.44$ in the correlational model. Results of the instability analyses at the between-person level can be found in Table 3. PNI grandiosity was significantly positively associated with SSD grandiosity but was not significantly associated with SSD vulnerability. PNI vulnerability, on the other hand, was not significantly associated with SSD grandiosity but was significantly correlated with SSD vulnerability. This significant associations disappeared once accounting for shared variance in the predictors.

Table 3. Zero order and regression paths of instability at the between-person level

\begin{tabular}{|c|c|c|c|c|}
\hline & \multicolumn{4}{|c|}{ Squared Difference } \\
\hline & \multicolumn{2}{|c|}{ Grandiosity } & \multicolumn{2}{|c|}{ Vulnerability } \\
\hline & $r$ & $\beta$ & $r$ & $\beta$ \\
\hline PNI Grandiosity & $.16[.03, .30]$ & $.21[.05, .37]$ & $.12[-.02, .26]$ & $.07[-.06, .19]$ \\
\hline PNI Vulnerability & $-.00[-.13, .12]$ & $-.08[-.22, .13]$ & $.20[.03, .34]$ & $.16[.-.01, .31]$ \\
\hline
\end{tabular}

\section{Inertia}

Results from calculations at the within-person level for inertia can be found in Figure 5. Previous grandiose $\left(\mathrm{NG}_{\mathrm{t}-1}\right)$ states moderately predicted current grandiosity states $\left(\mathrm{NG}^{*} \mathrm{t}\right)$ and modestly predicted current vulnerability states $\left(\mathrm{NV} *_{\mathrm{t}}\right)$. Previous vulnerability states $\left(\mathrm{NV} *_{\mathrm{t}-1}\right)$ moderately predicted current vulnerable states and did not predict current grandiosity states. At 
the between-person level, individual differences in inertia were regressed on dispositional grandiosity and vulnerability (see Table 4). Only dispositional vulnerability was associated with the within-person means of vulnerability $\left(\mu_{\mathrm{NV}}\right)$.

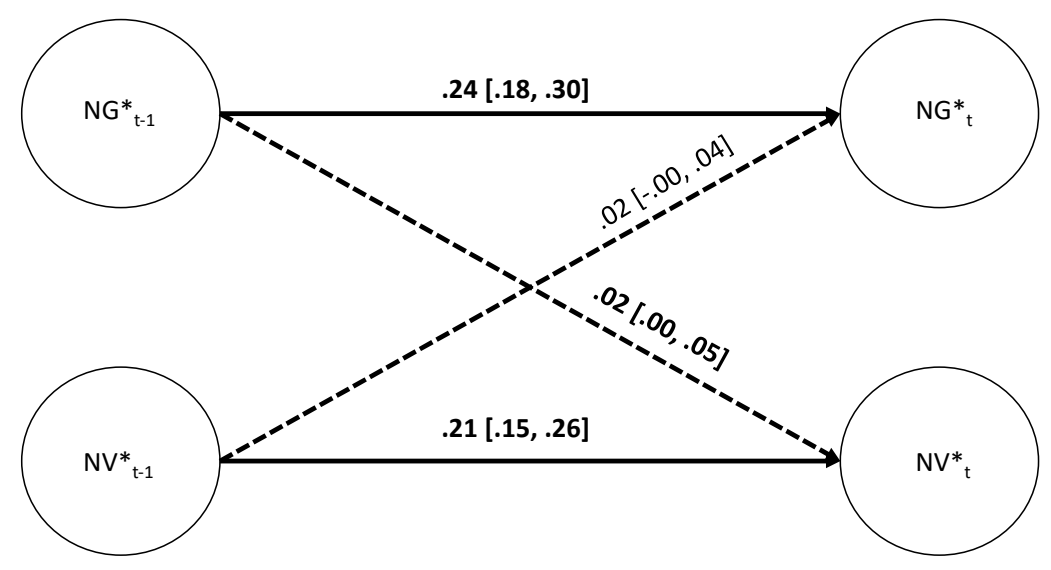

Figure 5. $\mathrm{N}=231$. $\mathrm{NG}=$ Narcissistic Grandiosity; $\mathrm{NV}=$ Narcissistic Vulnerability. Within-person results of inertia coefficients. Bolded values are those for which the credibility interval did not contain zero. Dotted lines are those for which the predicted domain.

Table 4. Results of multilevel regression results of inertia at the between person level

\begin{tabular}{lcccccc}
\hline & $\varphi_{\mathrm{G} \rightarrow \mathrm{G}}$ & $\varphi_{\mathrm{V} \rightarrow \mathrm{V}}$ & $\varphi_{\mathrm{V} \rightarrow \mathrm{G}}$ & $\varphi_{\mathrm{G} \rightarrow \mathrm{V}}$ & $\mu_{\mathrm{NG}}$ & $\mu_{\mathrm{NV}}$ \\
\cline { 2 - 7 } PNI-G & $-.13[-.27, .02]$ & $-.02[-.14, .08]$ & $.04[-.15, .23]$ & $.07[-.08, .22]$ & $.06[-.03, .16]$ & $-.01[-.09, .10]$ \\
PNI -V & $-.02[-.17, .08]$ & $.09[-.06, .22]$ & $-.13[-.37, .06]$ & $-.04[.18, .09]$ & $-.09[-.18, .01]$ &. $\mathbf{1 1}[.03, .22]$ \\
\hline
\end{tabular}

Note. $N=231$. Bolded values are those for which the credibility interval did not contain zero. Values on the right predicted column headings. PNI=Pathological Narcissism Inventory. 


\section{Switching}

The present study took a relatively strict approach to coding switches. As such, only 115 observations (out of 7,480) were characterized as a switch.

\section{Switching Propensity}

Results for switching propensity can be found in Table 5. Firstly, I was interested in whether individuals switched in any direction (Any Switch). This included switching within a domain and switching across domains. Results revealed that dispositional grandiosity modestly negatively predicted any form of switching. Neither dispositional vulnerability or grandiosity were predictive of switching across domains. Next, of interest was whether dispositional scores were predictive of switching in a specific direction (i.e., from grandiosity to vulnerability or from vulnerability to grandiosity). Dispositional narcissism scores were non-significantly associated with movement in a specific direction. Finally, I examined whether dispositional scores were predictive of switching within a state (e.g., above threshold for grandiosity to below). Dispositional grandiosity was modestly positively predictive of switching within grandiosity. Dispositional vulnerability was moderately negatively associated with switching within grandiosity and was moderately positively associated with switching within vulnerability.

Table 5. Switching propensity at the between-person level

\begin{tabular}{|c|c|c|c|c|c|c|}
\hline & Any Switch & $\begin{array}{c}\text { Across } \\
\text { Domains }\end{array}$ & To Grandiose & To Vulnerable & $\begin{array}{c}\text { Within } \\
\text { Grandiosity }\end{array}$ & $\begin{array}{c}\text { Within } \\
\text { Vulnerability }\end{array}$ \\
\hline $\begin{array}{l}\text { PNI } \\
\text { Grandiosity } \\
\text { PNI }\end{array}$ & $-.18[-.36,-.01]$ & $.01[-.19, .24]$ & .05 [-.31,.36] & $.01[-.26, .26]$ & $.16[.01, .27]$ & $-.10[-.26, .05]$ \\
\hline Vulnerability & $.00[-.19, .16]$ & $.04[-.19, .24]$ & $.15[-.19, .46]$ & $.05[-.21, .29]$ & $-.26[-.38,-.13]$ & $.29[.13, .43]$ \\
\hline
\end{tabular}




\section{Switching Distance}

Results examining switching distance in consecutive grandiose and vulnerable states were examined as a function of switch and no switch can be found in Table 6. At level 1, switching distance was modeled as a function of switch and no switch groups. No switch and switch groups were both significant predictors of switching distance for both grandiosity and vulnerability, with stronger effects for the switch condition in each group. At level 2, dispositional scores were used to predict switching distance for switch and no switch groups. Only dispositional grandiosity was a significant predictor of switching distance for the no switching group. This was true in both the grandiose and vulnerable condition. 
Table 6. Switching distance across switch and no switch for grandiosity and vulnerability

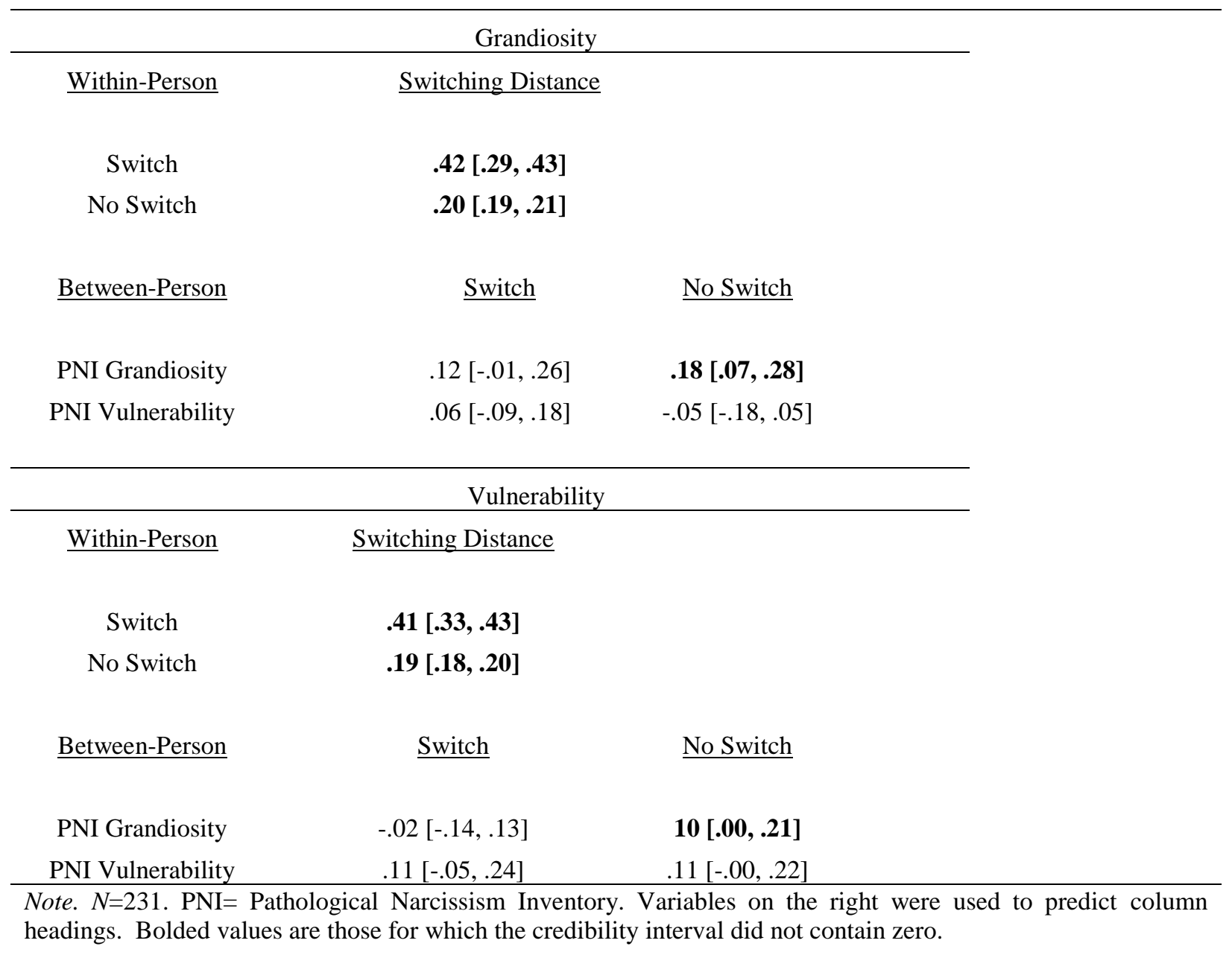

\section{Switching Distance with Correction for Starting Point of a Switch}

The next step is to understand associations between switches and non-switches once accounting for overall switching distance. Results of switching distance within categories, once accounting for the starting point can be found in Table 7. Examination of results suggest that accounting for overall switching distance within grandiosity and vulnerability does not change the results substantially. In particular, similar results emerge (Table 7) to those in Table 6. The main exception is that dispositional grandiosity no longer remains a significant predictor of variability within the no switch group for vulnerability. 
Table 7. Switching distance across switch and no switch for grandiosity and vulnerability with correction of starting point

\begin{tabular}{|c|c|c|}
\hline \multicolumn{3}{|c|}{ Grandiosity } \\
\hline Within-Person & $\underline{\text { Switching Distance }}$ & \\
\hline Switch & $.39[.27, .40]$ & \\
\hline No Switch & $.18[.17, .19]$ & \\
\hline Between-Person & $\underline{\text { Switch }}$ & No Switch \\
\hline PNI Grandiosity & $.12[-.00, .23]$ & $.13[.03, .22]$ \\
\hline PNI Vulnerability & $.05[-.08, .16]$ & $.01[-.10, .12]$ \\
\hline \multicolumn{3}{|c|}{ Vulnerability } \\
\hline$\underline{\text { Within-Person }}$ & $\underline{\text { Switching Distance }}$ & \\
\hline Switch & $.38[.09, .39]$ & \\
\hline No Switch & $.18[.13, .19]$ & \\
\hline$\underline{B e t w e e n-P e r s o n}$ & $\underline{\text { Switch }}$ & No Switch \\
\hline PNI Grandiosity & $-.06[-.19, .08]$ & $.07[-.02, .21]$ \\
\hline PNI Vulnerability & $.06[-.07, .20]$ & $.03[-.08, .13]$ \\
\hline
\end{tabular}




\section{Switch Distance in Different Directions ${ }^{1}$}

Results examining switching in specific direction of the switch (e.g., from above threshold to below threshold) for each domain can be found in Table 8. At level 1, for both grandiosity and vulnerability, all possible conditions (i.e., switching in to a state, switching out of a state, and no switch) were modest significant predictors of overall switching distance. In the grandiose condition at level 2, dispositional grandiosity was a significant predictor of switching distance in the no switch group. In the vulnerable condition, dispositional vulnerability emerged as a modest predictor of switching distance for those that involved switches from above threshold for vulnerability to below and was a modest predictor of switching distance for the no switch group.

${ }^{1}$ The present results were run without correcting for the starting point of consecutive states. Additional analyses suggest that correcting for the starting point does not change significance at level 1. At level 2, dispositional scores become non-significant predictors of switching distance in any switching category for either grandiosity or vulnerability. 


\subsection{Discussion}

Clinicians have noted that narcissistic individuals fluctuate in their expressions of grandiosity and vulnerability (e.g., Kernberg, 1975; Wright, 2014; Pincus et al., 2014; Ronningstam, 2009, 2011). Consistent with clinical theory, emerging empirical evidence is suggestive of dynamic processes within and between grandiosity and vulnerability (e.g., Gore \& Widiger, 2016; Giacomin \& Jordan, 2016). However, no study has directly examined these two components using methodology specifically designed to capture the theorized dynamic processes. More specifically, studies have either not examined this question with regard to grandiosity and vulnerability (e.g., Geukes et al., 2016) or have continued to use trait measures to understand this process (e.g., Gore \& Widiger, 2016).

Clinical descriptions of variability within and between grandiose and vulnerable states have not detailed the nature or duration of these patterns. Coupled with a dearth of research systematically examining these processes, little a priori information is available about the timescale and nature of these fluctuations. Accordingly, I adopted a descriptive and exploratory approach towards investigating fluctuations in grandiosity and vulnerability. The first aim of my study was to elucidate how grandiosity and vulnerability varied moment to moment, focusing on continuous fluctuation in each dimension independently of the other. The second aim was to examine the interplay among these two domains, or how grandiosity and vulnerability related to each other within individuals over time. As an adjunct to this question, I examined movement in and out of states within individual domains over time. Further, to provide an understanding of how variability on these momentary assessments of narcissism relate to dispositionally measured narcissism, I examined associations with the PNI. 
The first set of analyses in the current study examined fluctuations in dimensional measures of variability. Fluctuation was operationalized in three ways: gross variability, instability, and inertia. Gross variability was the only metric that did not hinge strongly on the specific ordering of time (i.e., successive observations separated by $\geq 90$ minutes). Due to the exploratory nature of this study, operationalizations of variability that were unstructured across time arguably provided the most information. The other operationalizations of variability, instability and inertia, as well as explicit switching between states, are directly tethered to the ordering of assessments, and are, thus, more strongly impacted by the timescale. Although statistical techniques that allow for the comparison of one moment to the next are useful and necessary as research in narcissism seeks to pin down fluctuations, they require an understanding of time as it pertains to the variables of interest. In line with this, the results pertaining to gross variability are likely the most relevant for understanding the overall nature of fluctuations in momentary grandiosity and vulnerability and will be the primary focus in the discussion, with results for instability and inertia treated as providing supplementary information.

Closer examination of the gross variability findings suggests that, in line with my hypotheses, individuals with higher mean levels of momentarily-assessed grandiosity or vulnerability tend to fluctuate more in the respective domain. These data demonstrated somewhat of a floor effect, such that individuals with higher levels of grandiosity or vulnerability had more room to vary within either domain. This finding is consistent with previous research that has found associations between mean levels and overall fluctuations (e.g., Baird, Le, \& Lucas, 2006). As noted above, some have interpreted this pattern as an artifact of higher mean levels; people who have higher values of grandiosity or vulnerability by definition vary more. Alternatively, substantive reasons could account for the association between mean and variance observed here. 
For instance, it may be that individuals who are characteristically higher in a given domain become more volatile and it becomes difficult to maintain high levels of grandiosity or vulnerability. Another possibility is that the skewed distribution reflects "flare ups" of elevated scores, leading to higher means for those who experience more of these events. In these two examples, the link between mean level and variability would not be considered an artefactual association but rather reflective of a specific process unfolding. Disambiguating artefactual from substantive interpretations of mean and variance covariation goes well beyond this study's scope, and, in fact, remains a topic of debate in the literature (e.g., Mestdagh et al., 2018).

Though not a goal of the present study to clarify differences between substantive and artifactual associations with momentary means and variability, it is important to understand the full extent to which momentarily-assessed means impact the results. Specifically, the present study also demonstrated interesting results regarding associations between dispositional narcissism and momentarily-assessed means. Individuals with higher dispositional grandiosity scores had higher momentary means in both grandiosity and vulnerability. The same was not true for individuals who were dispositionally vulnerable. Individuals with higher dispositional vulnerability scores exhibited lower momentary grandiosity averages. This finding is similar to those in Gore and Widiger (2016) and Hyatt and colleagues (2017). Specifically, they showed that grandiose individuals may experience bouts of vulnerability in addition to grandiosity. Vulnerable individuals, on the other hand, were not shown to have higher mean levels of grandiosity. Given that associations with overall mean levels appear to play a key role in the analyses of the present study, following other authors (e.g., Eid \& Diener, 1999), where relevant I examined associations between dispositional scores and overall variability, both with and without adjusting for overall mean levels of the outcome. 
It was hypothesized that across all three forms of overall variability, dispositional vulnerability would be associated with relative instability (i.e., higher gross variability and instability values, and lower inertia values) in both grandiosity and vulnerability. This hypothesis was partially supported. Indeed, in analyses of gross variability, vulnerability tended to be a robust predictor of overall variability within vulnerability. Effects between dispositional vulnerability and overall variability emerged both as a pathway through mean level vulnerability and as a direct effect, once accounting for mean level. Given the maintained effect after accounting for the mean, this suggests that the association between dispositional vulnerability and variability in momentary vulnerability is not solely artifactual, but rather suggests individuals with higher dispositional vulnerability scores are subject to greater variability in vulnerability over time. This conclusion was further supported with moderate correlations between dispositional vulnerability and instability in vulnerability. Research has suggested that vulnerability tends to be a measure of distress and is associated with a wide range of pathologies (e.g., Miller et al., 2014, 2016; Edershile et al., in press; Pincus \& Lukowitsky, 2010). Indeed, previous literature has found associations between various other forms of distress (e.g., depression and negative affect) and overall variability (Houben et al., 2015).

Contrary to my hypothesis, dispositional vulnerability did not emerge as a predictor of gross variability in grandiosity, once accounting for the mean in momentary grandiosity. Nor was dispositional vulnerability a significant predictor of instability or inertia in grandiosity. Resulting evidence continues to suggest that dispositionally vulnerable individuals are not reaching high levels of grandiosity. Only through the path of mean level vulnerability in gross variability did a significant path emerge, such that more vulnerable individuals experienced more variability in grandiosity. Given that this pathway only emerged once accounting for the mean, interpretations 
must be made with caution. However, it is possible that, though they do not experience high levels of grandiosity, individuals with higher vulnerability do in fact vary in grandiosity. Whereas vulnerability has been shown to be a measure of distress, grandiosity has been shown to map on to a variety of adaptive features (Brown \& Ziegler-Hill, 2004; Morf \& Rhodewalt, 2001). Thus, it may be that individuals who are dispositionally vulnerable do not tend to experience higher grandiosity, per se, though what grandiosity they do experience is likely to be unstable across time. This expands on the previous work by Gore and Widiger (2016) and Hyatt and colleagues (2017) to suggest that while individuals who are dispositionally vulnerable may not have high levels of grandiosity, they do vary within this domain.

I additionally hypothesized that dispositional grandiosity scores would tend to be associated with relative stability (i.e., lower gross variability and instability, and higher inertia values) in both grandiosity and vulnerability. This hypothesis was not supported. Individuals dispositionally high in grandiosity tended to have more momentary variability in grandiosity, but only in the pathway through the overall mean level. The direct path from dispositional grandiosity to variability in grandiosity was non-significant after accounting for the mean. Again, this leaves some ambiguity regarding the substantive versus artifactual nature of these findings, but it can be cautiously suggested that individuals high in dispositional grandiosity vary more in their levels of grandiosity. Indeed, higher dispositional scores were associated with instability in momentary grandiosity. This is contrary to previous research that suggests that grandiosity tends to be associated with higher and more stable self-esteem (e.g., Geukes et al., 2017; Carlson, Vazire, \& Oltmanns, 2011). Individuals with high levels of grandiosity may experience bouts of high selfesteem but also may have episodes of vulnerability, making fluctuations in both domains likely. Indeed, dispositional grandiosity was associated with variability in vulnerability, indirectly 
through the mean of vulnerability. Summarizing overall variability, the present results expand on previous research to suggest that while grandiose individuals may have bouts of both grandiosity and vulnerability and vulnerable individuals do not appear to experience high levels of grandiosity, both individuals are prone to experience variability within and across domains.

Moving past continuous articulations of variability, explicit switching between states of grandiosity and vulnerability was examined. It was hypothesized that dispositional grandiosity would be related to a lower frequency of switching whereas vulnerability would be related to a higher rate of switching, as measured through a variety of analytic strategies. My hypotheses were not supported. In terms of switching across domains, few significant results emerged, such that neither dispositionally grandiose or vulnerable individuals were likely to switch across domains. The lack of associations emerging here suggests that if these switches do occur, they do not occur on the timescale assessed in the present study (i.e., 90 minutes). Indeed, patient observation suggests these fluctuations may occur from one week to the next or perhaps longer (e.g., Wright, 2014). Thus, as researchers seek to align clinical theory and empirical evidence, it will be important to continue the exploratory nature used in this study and include broader assessment timescales.

In addition to switching across domains, it was examined if individuals had a propensity to switch within a given domain. Though clinicians have theories directly relating to switching between states of grandiosity and vulnerability (e.g., Wright, 2014; Pincus, Cain, \& Wright, 2014; Ronningstam, 2009, 2011), it is possible that individuals also switch in and out of states within a given domain. Though it was predicted that dispositional grandiosity would be related to a lower likelihood to switch whereas vulnerability would be related to a greater likelihood, results did not reveal this to be the case. Dispositional grandiosity scores were related to switching within 
grandiosity. Dispositional vulnerability scores were moderately negatively associated with switching within grandiosity and were positively associated with switching within vulnerability. Though contrary to what was hypothesized, this is in line with the previous results of overall variability. Similar to findings that emerged in gross variability, dispositionally vulnerable individuals do not appear to experience levels of grandiosity that reach such extremes to be characterized as a "state of grandiosity." These same individuals do come in and out of states of vulnerability, however. Individuals high in dispositional grandiosity also come in and out of states of grandiosity, but do not appear to follow as clear of a pattern with vulnerability. In the present study, criteria for "states" hinged on group means and it has been repeatedly demonstrated here that different subtypes of narcissism reach different levels in each domain, on average. Thus, future research wishing to further examine switching between grandiosity and vulnerability should critically consider mean differences in dispositionally grandiose and dispositionally vulnerable individuals.

The final set of analyses examined whether switching distances differed between instances of switching and not switching. Dispositional scores were again used to predict these changes. My hypotheses were unsupported. Switching and non-switching occasions were both positively associated with overall switching distance. Using dispositional scores to predict overall distance did not further elucidate these findings, such that no clear descriptive picture emerged. This may be a function of how switching was defined. Taking a relatively strict definition of switching (i.e., the individual had to be above threshold in the prior assessment in one domain and below in the other, then switch in the current assessment) allowed for only $1.5 \%$ of observations to be characterized as a switch. Future research may want to perform the same analyses with less strict cutoffs for a switch. Further, there is a large number of reasons to use dimensional scores to 
understand psychological processes. For example, many would argue that dimensions create a more realistic and useful approach to capturing how personality pathology manifests (e.g., Krueger et al., 2011). However, in this undergraduate sample, with such small numbers meeting switching criteria, it may be that additionally having very few participants on the extreme ends of the narcissism spectrum compounds the difficulty of observing any effects with switching. Of note, previous research examining switching patterns in other disorders (e.g., borderline personality disorder) have used diagnosis or an extreme case approach as a predictor rather than dimensional scores (Houben et al., 2016).

Overall, the manner in which data was captured in the present study (including the timescale, the strict switching criteria, and non-clinical sample) ultimately complicated the narrative for two out of the three measures of continuous measures of variability and did not lend itself to understanding the switching dynamics between grandiosity and vulnerability that have been clinically observed (e.g., Wright 2014; Pincus et al., 2014; Ronningstam, 2009, 2011). However, results emerge that suggest important differences between individuals who are dispositionally grandiose and dispositionally vulnerable. Indeed, previous research (e.g., Gore \& Widiger, 2016; Hyatt et al., 2017) has demonstrated that grandiose individuals experience bouts of vulnerability whereas vulnerable individuals do not experience periods of grandiosity in the same way. Results of the present study largely replicated these findings. However, it was additionally found that while dispositionally vulnerable individuals tend to have higher mean levels of vulnerability and lower mean levels of grandiosity, they do show substantial variability in each domain. Dispositionally grandiose individuals tend to have higher means of both grandiosity and vulnerability across time and also vary in each. Thus, while overall mean levels of grandiosity and vulnerability may be a discriminating feature across narcissistic individuals, 
variability is a key component of the processes within individuals. This has important implications for future research as researchers begin to consider the timescale, importance of controlling for mean level, and the overall nature of switching dynamics within narcissism. Though not elucidating specific switching patterns, per se, this study sheds light on important dynamic associations that will be crucial as researchers work to further align empirical evidence with clinical theory of fluctuations between the two core domains of narcissism.

\subsection{Limitations and Future Directions}

The present study used an undergraduate sample as the population. Indeed, research on narcissism overwhelming uses undergraduate samples. Nonetheless, research in other areas used clinical and community samples to address questions similar to this one (e.g., Houben et al., 2015; 2016). Further, given the results of the present study suggest that specific dynamics of narcissism may be better observed in more pathological individuals, it would behoove future researchers to design a study specifically targeting a more pathological population.

Additionally, this study used the PNI as the dispositional measure of comparison. Previous work has demonstrated that the grandiosity scale of the PNI tends to be saturated with vulnerability (Miller et al., 2014; 2016). Other work has shown that true grandiose themes emerge when accounting for shared variance with vulnerability (Edershile et al., in press), as they did here in multilevel regression paths. Accordingly, when using this measure, it is best for analyses to be run from both a correlational and regression perspective, as was done in the present study, to understand the full extent of associations. Future work should continue to explore this research 
question and include other dispositional measures that have been shown to have strong associations with momentary narcissism assessments (Edershile et al., under review).

Given that the present study provided initial evidence of unique patterns of variability for individuals who are dispositionally grandiose versus dispositionally vulnerable, future directions include elaborating on the nature of this variability. In this study, thresholds for switching were limited by the random two-hour sampling employed. Additionally, "states" were those scores above the grand mean of an individual domain. These thresholds contributed to a particularly low number of individuals who met criteria for a "switch". It may be that additional information is needed before switching criteria is determined. In particular, now that it is known that systematic variability within narcissism exists, situational contexts that may drive an individual up and down within these domains should be examined. Preliminary research suggests that responses to different interpersonal contexts look vastly different for individuals considered dispositionally grandiose compared to dispositionally vulnerable individuals (Edershile \& Wright, in preparation). Having samples uniquely geared towards more narcissistic individuals coupled with an interest in the context of overall variability should perhaps serve as the gold standard for researchers wishing to understand the complex processes within narcissism. It will be important to engage both of these strategies with a range of different sampling timeframes (e.g., monthly, weekly, daily). Broadly, elucidating specific switching dynamics may not be the key to understanding momentary manifestations of narcissism. Rather, the answer may lie in gaining information of the contexts in which highly narcissistic individuals vary. 


\subsection{Conclusion}

Research in the field of narcissism has suggested a dynamic process between two components of narcissism: grandiosity and vulnerability. Pinning down these processes is quickly becoming the most pressing question in the field. The present study was the first to my knowledge to explicitly examine this dynamic process between grandiosity and vulnerability from a momentary perspective. A variety of different statistical approaches that have been used to study other personality pathologies were engaged to examine these theorized processes. This study demonstrated key distinctions with regard to momentary patterns of vulnerable and grandiose individuals who will be important to consider as the field moves to further examine the complex dynamics of narcissism. Though few significant results emerged with regard to switching between

states, the present study elucidated important dynamics of overall variability, giving the field a framework for how it is possible to examine differences between grandiose and vulnerable narcissistic states. As such, this study serves as an important stepping stone as researchers work to better understand the dynamic processes occurring within narcissism. 


\section{Bibliography}

Akhtar, S., \& Thomson, J. A. (1982). Overview: Narcissistic personality disorder. American Journal of Psychiatry, 139, 12-20.

American Psychiatric Association. (2013). Diagnostic and statistical manual of mental disorders ( $5^{\text {th }}$ ed.). Washington D.C.: Author.

American Psychiatric Association. (1980). Diagnostic and statistical manual of mental disorders ( ${ }^{\text {rd }}$ ed.). Washington D.C.: Author.

Aslinger, E.N., Manuck, S.B., Pilkonis, P.A., Simms, L.J., \& Wright, A.G.C. (2018). Narcissist or narcissistic? Evaluation of latent structure of narcissistic personality disorder. Journal of Abnormal Psychology, 127(5), 496-502.

Asparouhov, T., Hamaker, E. L., \& Muthén, B. (2017). Dynamic latent class analysis. Structural Equation Modeling, 24, 257-269.

Asparouhov, T., Hamaker, E. L., \& Muthén, B. (2018). Dynamic structural equation models. Structural Equation Modeling, 25, 359-388.

Back, M. D., Küfner, A. C. P., Dufner, M., Gerlach, T. M., Rauthmann, J. F., \& Denissen, J. J. A. (2013). Narcissistic admiration and rivalry: Disentangling the bright and dark sides of narcissism. Journal of Personality and Social Psychology, 105, 1013-1037.

Baird, B.M., Le, K., \& Lucas, R.E. (2006). On the nature of intraindividual personality variability: reliability, validity, and associations with well-being. Journal of Personality and Social Psychology, 90 (3), 512-527.

Besser, A., Zeigler-Hill, V. (2010). The influence of pathological narcissism on emotional and motivational responses to negative events: The roles of visibility and concern about humiliation. Journal of Research in Personality, 44, 520-524.

Bosson, J. K., Lakey, C. E., Campbell, W. K., Zeigler-Hill, V., Jordan, C. H., \& Kernis, M. H. (2008). Untangling the links between narcissism and self-esteem: A theoretical and empirical review. Social and Personality Psychology Compass, 2, 1415-1439.

Brown, R. P., \& Ziegler-Hill, V. (2004). Narcissism and the non-equivalence of self-esteem measures: A matter of dominance? Journal of Research in Personality, 38, 585-592.

Buss, D.M., \& Chiodo, L.M. (1991). Narcissistic acts in everyday life. Journal of Personality, 59, 179-215. 
Cain, N.M, Pincus, A.L., \& Ansell, E.B. (2008). Narcissism at the crossroads: Phenotypic description of pathological narcissism across clinical theory, social/personality psychology, and psychiatric diagnosis. Clinical Psychology Review, 28, 638-656.

Carlson, E. N., Vazire, S., \& Oltmanns, T. F. (2011). You probably think this paper's about you: Narcissists' perceptions of their personality and reputation. Journal of Personality and Social Psychology, 101, 185-201.

Crowe, M., Carter, N.T., Campbell, K.W., \& Miller, J.D. (2016). Validation of the Narcissistic Grandiosity Scale and creation of item variants. Psychological Assessment, 28, 1550-1560.

Crowe, M. L., Edershile, E. A., Wright, A. G. C., Lynam, D. R., \& Miller, J. D. (2018). Development and validation of the Narcissistic Vulnerability Scale: An adjective rating scale. Psychological Assessment, 30(7), 978-983.

Dickinson, K. A., \& Pincus, A. L. (2003). Interpersonal analysis of grandiose and vulnerable narcissism. Journal of personality disorders, 17, 188 -207.

Edershile, E. A., Simms, L. J., \& Wright, A. G. C. (in press). A multivariate analysis of Pathological Narcissism Inventory's nomological network. Assessment.

Edershile, E.A., \& Wright, A.G.C. (2018, June). A momentary perspective of narcissism and interpersonal behavior perceptions. Paper to be presented at the annual meeting of the Society for Interpersonal Theory and Research, Montreal, Quebec.

Edershile, E.A., \& Wright, A.G.C. (in preparation). Narcissistic States and Interpersonal Situations. Journal of Self and Identity, Special Issue: "The Many Faces of Narcissism."

Edershile, E.A., Woods, W.C., Sharpe, B.M., Crowe, M.L., Miller, J.D., \& Wright, A.G.C. (under review). A day in the life of Narcissus: Measuring narcissistic grandiosity and vulnerability in daily life. Psychological Assessment.

Eid, M., Diener, E. (1999). Intraindividual variability in affect: Reliability, validity, and personality correlates. Journal of Personality and Social Psychology, 76(4), 662-676.

Gabbard, G.O. (2009). Transference and countertransference: developments in the treatment of narcissistic personality disorder. Psychiatric Annals, 39, 129-136.

Geukes, K., Nestler, S., Hutteman, R., Dufner, M., Kufner, A. C. P., Egloff, B., Denissen, J. J. A., $\&$ Back, M. D. (2016). Puffed-up but shaky selves: State self-esteem level and variability in narcissists. Journal of Personality and Social Psychology: Personality Processes and Individual Differences, doi.org/10.1037/pspp0000093

Giacomin, M., \& Jordan, C. H. (2016). Self-focused and feeling fine: Assessing state narcissism and its relation to well-being. Journal of Research in Personality, 63, 12-21. 
Glover, N., Miller, J.D., Lynam, D.R., Crego, C., \& Widiger, T.A. (2012). The five-factor narcissism inventory: A five-factor measure of narcissistic personality traits. Journal of Personality Assessment, 94(5), 500-512.

Gore, W. L., Widiger, T. A. (2016). Fluctuation between grandiose and vulnerable narcissism. Personality Disorders: Theory, Research, and Treatment, 7, 363-371.

Grubbs J.B., Exline, J.J (2016). Trait Entitlement: A cognitive-personality source of vulnerability to psychological distress. Psychological Bulletin, 142, 1204-1226.

Hendin, H.M., \& Cheek, J.M. (1997). Assessing Hypersensitive Narcissism: A Re-examination of Murray's Narcissism Scale. Journal of Research in Personality, 31, 588-599.

Houben, M., Van Den Noortgate, W., \& Kuppens, P. (2015). The relation between short-term emotion dynamics and psychological well-being: A meta-analysis. Psychological Bulletin, 141, 901-930.

Houben, M., Vansteelandt, K., Claes, L., Sienaert, P., Berens, A., Sleuwaegan, E., \& Kuppens, P. (2016). Emotional switching in Borderline Personality Disorder: A daily life study. Personality Disorders: Theory, Research, and Treatment, 7, 50-60.

Houlcroft, L., Bore, M., \& Munro, D. (2012). Three faces of narcissism. Personality and Individual Differences, 53, 274-278.

Hyatt, C.S., Sleep, C.E., Lynam, D.R., Widiger, T.A., Campbell, W.K., \& Miller, J.D. (2017). Ratings of affective and interpersonal tendencies differ for grandiose and vulnerable narcissism: a replication and extension of Gore and Widiger (2016). Journal of Personality, $1-13$.

Hyler, S. E. (1994). Personality Diagnostic Questionnaire-4 (PDQ-4). New York, NY: New York State Psychiatric Institute.

Kernberg, O. F. (1975). Borderline conditions and pathological narcissism. New York, NY: Aronson.

Krizan, Z., \& Herlache, A.D. (2017). The narcissism spectrum model: a synthetic review of narcissistic personality. Personality and Social Psychology Review, 22, 3-31.

Krueger, R.F., Eaton, N.R, Clark, Watson, D., Markon, K.E., Derringer, J., Skodol, A., \& Livesley, W.J. (2011). Deriving an empirical structure of personality pathology for DSM-5. Journal of Personality Disorders, 25(2), 170-191.

Levy, K.N, Reynoso, J.S, Wasserman, R.H, Clarkin, J.F. (2007). Narcissistic personality disorder. In W. O'Donohue, K.A Fowler, S.O Lilienfeld (Ed.) Personality Disorders: Toward the $D S M-V$ (pp. 233-77). Thousand Oaks, CA: Sage 
Mestdagh, M., Pe, M., Pestman, W., Verdonck, S., Kuppens, P., \& Tuerlinckx, F. (2018). Sidelining the mean: The relative variability index as a generic mean-corrected variability measure for bounded variables. Psychological Methods. Advance online publication.

Miller, J. D., Dir, A., Gentile, B., Wilson, L., Pryor, L. R., \& Campbell, W. K. (2010). Searching for a vulnerable dark triad: Comparing factor 2 psychopathy, vulnerable narcissism, and borderline personality disorder. Journal of Personality, 78, 1529-1564.

Miller, J. D., Hoffman, B. J., Gaughan, E. T., Gentile, B., Maples, J., \& Campbell, W. K. (2011). Grandiose and vulnerable narcissism: A nomological network analysis. Journal of Personality, 79, 1013-1042.

Miller, J. D., Lynam, D. R., \& Campbell, W. K. (2016). Measures of narcissism and their relations to DSM-5 pathological traits: a critical reappraisal. Assessment, 23, 3-9.

Miller, J. D., Lynam, D. R., Hyatt, C. S., \& Campbell, W. K. (2017). Controversies in narcissism. Annual Review of Clinical Psychology, 13, 1-54.

Miller, J.D., Lynam, D.R., McCain, J.L., Few, L.R., Crego, C., Widiger, T.A., \& Campbell, W.K. (2015). Thinking structurally about narcissism: an examination of the five-factor narcissism inventory and its components. Journal of Personality Disorder, 29, 1-18.

Miller, J. D., Lynam, D. R, McCain, J. L., Few, L. R., Crego, C., Widiger, T. A., Campbell, W. K.(2016c). Thinking structurally: A test of the factor structure of the Five-Factor Narcissism Inventory. Journal of Personality Disorders, 30, 1-18.

Miller, J. D., McCain, J., Lynam, D. R., Few, L. R., Gentile, B., MacKillop, J., Campbell, W. K. (2014). A comparison of criterion validity of popular measures of narcissism and Narcissistic Personality Disorder via the use of expert ratings. Psychological Assessment, 26, 958-969.

Morf, C.C. (2006). Personality reflected in a coherent idiosyncratic interplay of intra- and interpersonal self-regulatory processes. Journal of Personality, 76, 1527-1556.

Morf, C., \& Rhodewalt, F. (2001). Unraveling the paradoxes of narcissism: A dynamic selfregulatory processing model. Psychological Inquiry, 12, 177-196.

Morf, C.C., Schürch, E., Küfner, A., Siegrist, P., Vater, A., Back, M., \& Schröder-Abé, M. (2017). Expanding the nomological net of the Pathological Narcissism Inventory: German validation and extension in a clinical inpatient sample. Assessment, 24, 419-443.

Muthén, L. K., \& Muthén, B. O. (1998). Mplus User's Guide. (Eighth Edition.). Los Angeles, CA: Muthén \& Muthén.

Paulhus, D.L., \& Williams, K.M. (2002). The dark triad of personality: Narcissism, Machiavellianism, and psychopathy. Journal of Research in Personality, 36, 556-63. 
Pincus, A. L., Ansell, E. B., Pimentel, C. A., Cain, N. M., Wright, A. G. C., \& Levy, K. N. (2009). Initial construction and validation of the Pathological Narcissism Inventory. Psychological Assessment, 21, 265-279.

Pincus, A. L., Cain, N. M., \& Wright, A. G. C. (2014). Narcissistic grandiosity and narcissistic vulnerability in psychotherapy. Personality Disorders: Theory, Research, and Treatment, $1-5$.

Pincus, A. L., \& Lukowitsky, M. R., (2010). Pathological narcissism and narcissistic personality disorder. Annual Review of Clinical Psychology, 6, 421-446.

Raskin, R., \& Terry, H. (1988). A principal-components analysis of the Narcissistic Personality Inventory and further evidence of its construct validity. Journal of Personality and Social Psychology, 54(5), 890-902.

Rhodewalt, F., \& Morf, C. C. (1995). Self and interpersonal correlates of the Narcissistic Personality Inventory: A review and new findings. Journal of Research in Personality, 29, $1-23$.

Ronningstam, E. (2005). Narcissistic personality disorder: A review. In M. Maj, H. S. Akiskal, J. E. Mezzich, \& A. Okasha (Eds.), Personality disorders (pp. 277-327). New York, NY: Wiley.

Ronningstam, E. (2011). Narcissistic personality disorder: A clinical perspective. Journal of Psychiatric Practice, 17, 89-99.

Rosenthal, S.A., Hooley, J.M., Steshenko, Y. (2007). Distinguishing grandiosity from self-esteem: Development of the Narcissistic Grandiosity Scale. Unpublished Manuscript.

Schoenleber, M., Roche, M. J., Wetzel, E., Pincus, A. L., \& Roberts, B. W. (2015). Development of a brief version of the Pathological Narcissism Inventory. Psychological Assessment, 27(4), 1520-1526.

Thomas, K.M, Wright, A.G.C, Lukowitsky, M.R, Donnellan, M.B, Hopwood, C.J. (2012). Evidence for the criterion validity and clinical utility of the Pathological Narcissism Inventory. Assessment, 19, 135-45.

Wang, L., Hamaker, E., \& Bergeman, C. S. (2012). Investigating Inter-Individual Differences in Short-Term Intra-Individual Variability. Psychological Methods, 17(4), 567-581.

Watson, D., Clark, L. A., \& Tellegen, A. (1988b). Development and validation of brief measures of positive and negative affect: The PANAS Scales. Journal of Personality and Social Psychology, 47, 1063-1070.

Wink, P. (1991). Two faces of narcissism. Journal of Personality and Social Psychology, 61, 590597. 
Wright, A. G. C. (2014). Integrating trait and process based conceptualizations of pathological narcissism in the DSM-5 era. In A. Besser (Ed.), Handbook of psychology of narcissism: Diverse perspectives (pp. 153-174). Hauppauge, NY: Nova Science Publishers.

Wright. A.G.C. (2014). Narcissism and its discontents. Personality Disorders: Theory, Research, and Treatment, 5, 232-233.

Wright, A.G.C. (2016). On the Measure and Mismeasure of Narcissism: A Response to "Measures of narcissism and their relations to DSM-5 pathological traits: A critical re-appraisal." Assessment, 23, 10-17.

Wright, A. G. C., Lukowitsky, M.R., Pincus, A. L., \& Conroy, D.E. (2010). The higher order factor structure and gender invariance of the Pathological Narcissism Inventory. Assessment, 17, 467-483.

Wright, A.G.C., Pincus, A.L., Thomas, K.M., Hopwood, C.J., Markon, K.E., \& Krueger, R.F. (2013). Conceptions of narcissism and the DSM-5 pathological traits. Assessment, 20, 339352.

Wright, A. G. C., Simms, L. J. (2016). Stability and fluctuation of Personality Disorder features in daily life. Journal of Abnormal Psychology, 125, 641-656 